\title{
V-CYCLE OPTIMAL CONVERGENCE FOR CERTAIN (MULTILEVEL) STRUCTURED LINEAR SYSTEMS*
}

\author{
ANTONIO ARICÒ ${ }^{\dagger}$, MARCO DONATELLI ${ }^{\ddagger}$, AND STEFANO SERRA-CAPIZZANO ${ }^{\dagger}$
}

Abstract. In this paper we are interested in the solution by multigrid strategies of multilevel linear systems whose coefficient matrices belong to the circulant, Hartley, or $\tau$ algebras or to the Toeplitz class and are generated by (the Fourier expansion of) a nonnegative multivariate polynomial $f$. It is well known that these matrices are banded and have eigenvalues equally distributed as $f$, so they are ill-conditioned whenever $f$ takes the zero value; they can even be singular and need a low-rank correction.

We prove the V-cycle multigrid iteration to have a convergence rate independent of the dimension even in presence of ill-conditioning. If the (multilevel) coefficient matrix has partial dimension $n_{r}$ at level $r, r=1, \ldots, d$, then the size of the algebraic system is $N(n)=\prod_{r=1}^{d} n_{r}, O(N(n))$ operations are required by our technique, and therefore the corresponding method is optimal.

Some numerical experiments concerning linear systems arising in applications, such as elliptic PDEs with mixed boundary conditions and image restoration problems, are considered and discussed.

Key words. circulant, Hartley, and $\tau$ algebra, Toeplitz class, two-grid and multigrid iterations, multi-iterative methods, multilevel matrices

AMS subject classifications. $65 \mathrm{~F} 10,65 \mathrm{~F} 15,15 \mathrm{~A} 12$

DOI. $10.1137 /$ S0895479803421987

1. Introduction. Let $f(x), x=\left(x_{1}, \ldots, x_{d}\right)$, be a continuous function on the $d$-dimensional Euclidean space $\mathbb{R}^{d}$, and let $\langle\cdot \mid \cdot\rangle$ denote the usual scalar product between vectors. Henceforth, we suppose that $f$ has period $2 \pi$ with respect to each variable and is real valued, so the Fourier coefficients of $f$,

$$
a_{j}=\frac{1}{(2 \pi)^{d}} \int_{[-\pi, \pi]^{d}} f(x) e^{-\mathbf{i}\langle j \mid x\rangle} d x, \quad \mathbf{i}^{2}=-1, \quad j=\left(j_{1}, \ldots, j_{d}\right) \in \mathbb{Z}^{d},
$$

enjoy the relation $a_{-j}=\bar{a}_{j}$ for every $j \in \mathbb{Z}^{d}$. From the coefficients $a_{j}$ one can build [35] the sequence $\left\{T_{n}(f)\right\}, n=\left(n_{1}, \ldots, n_{d}\right) \in \mathbb{N}^{d}$, of multilevel Toeplitz matrices of size $N(n)=\prod_{r=1}^{d} n_{r}$. Every matrix $T_{n}(f)$ is explicitly written as

$$
T_{n}(f)=\sum_{|j| \leqslant n-e} a_{j} J_{n}^{[j]}=\sum_{\left|j_{1}\right| \leqslant n_{1}-1} \cdots \sum_{\left|j_{d}\right| \leqslant n_{d}-1} a_{\left(j_{1}, \ldots, j_{d}\right)} J_{n_{1}}^{\left[j_{1}\right]} \otimes \cdots \otimes J_{n_{d}}^{\left[j_{d}\right]} .
$$

Here $\otimes$ denotes the usual tensor product, so that $A \otimes B$ is the block matrix $\left[a_{i j} B\right]_{i j}$, $e=(1, \ldots, 1) \in \mathbb{N}^{d}$ and the relations between two multi-indices (as $|j| \leqslant n-e$ ) should be intended componentwise. If $n$ and $j$ are integer numbers, then $J_{n}^{[j]} \in \mathbb{R}^{n \times n}$ is the matrix whose entry $(s, t)$ equals 1 if $s-t=j$ and is 0 elsewhere; in the case where $n$ and $j$ are multi-indices, the symbol $J_{n}^{[j]}$ denotes the tensor product of all the $J_{n_{r}}^{\left[j_{r}\right]}$ for $r=1, \ldots, d$. From the identity $a_{-j}=\bar{a}_{j}$ for every $j$, it follows that the matrices

* Received by the editors January 31, 2003; accepted for publication (in revised form) by L. Reichel September 19, 2003; published electronically August 27, 2004.

http://www.siam.org/journals/simax/26-1/42198.html

†Dipartimento di Matematica "Felice Casorati," Università di Pavia, Via Ferrata 1, 27100, Pavia, Italy (arico@dimat.unipv.it, arico@dm.unipi.it).

${ }_{\ddagger}$ Dipartimento di Chimica, Fisica e Matematica, Università dell'Insubria-Sede di Como, Via Valleggio 11, 22100 Como, Italy (marco.donatelli@uninsubria.it, stefano.serrac@uninsubria.it, serra@mail.dm.unipi.it). 
$T_{n}(f)$ are Hermitian for every $n$. It is clear that, if $f$ is a trigonometric polynomial of degree $c=\left(c_{1}, \ldots, c_{d}\right)$, then the Fourier coefficient $a_{j}$ equals zero when $|j| \leqslant c$ is not satisfied; in that case the corresponding matrix $T_{n}(f)$ shows a $d$-level structure with bandwidth $c_{r}$ at level $r \in\{1, \ldots, d\}$.

To the same coefficients $a_{j}$ in (1.1) we can also associate matrices belonging to well-known trigonometric (multilevel) algebras. For instance, the $d$-level circulant related to $f$ is defined as

$$
\mathcal{C}_{n}(f)=\sum_{|j| \leqslant n-e} a_{j} Z_{n}^{[j]}=\sum_{\left|j_{1}\right| \leqslant n_{1}-1} \ldots \sum_{\left|j_{d}\right| \leqslant n_{d}-1} a_{\left(j_{1}, \ldots, j_{d}\right)} Z_{n_{1}}^{j_{1}} \otimes \cdots \otimes Z_{n_{d}}^{j_{d}},
$$

where $Z_{n}=J_{n}^{[-1]}+\mathbf{e}_{n} \mathbf{e}_{1}^{t}$ if $n$ is a scalar and $\mathbf{e}_{j}$ denotes the $j$ th vector of the canonical basis. Analogously to the Toeplitz case, if $n$ and $j$ are multi-indices, then $Z_{n}^{[j]}$ represents the tensor product of all the $Z_{n_{r}}^{j_{r}}$ for $r=1, \ldots, d$. If $f$ is even (with regard to each variable $x_{r}$ separately) we have $a_{j}=a_{-j} \in \mathbb{R}$, i.e., $T_{n}(f)$ is real and symmetric. In that case an interesting matrix algebra approximation is provided by the $\tau$ algebra [3]. More specifically we define

$$
\tau_{n}(f)=\sum_{0 \leqslant j \leqslant n-e} b_{j} H_{n}^{[j]}=\sum_{0 \leqslant j_{1} \leqslant n_{1}-1} \ldots \sum_{0 \leqslant j_{d} \leqslant n_{d}-1} b_{\left(j_{1}, \ldots, j_{d}\right)} H_{n_{1}}^{j_{1}} \otimes \cdots \otimes H_{n_{d}}^{j_{d}},
$$

where $H_{n}=J_{n}^{[1]}+J_{n}^{[-1]}$, the matrix $J_{n}^{[j]}$ is defined as before, and the coefficients $b_{j}$ can be uniquely determined by the coefficients $a_{j}$ through an invertible triangular linear system (see [24]). A further characterization of the $\tau$ algebra is obtained by observing that every matrix of the class can be written as a Toeplitz plus Hankel matrix (a Hankel matrix is constant along the antidiagonals): more precisely, we have

$$
\tau_{n}(f)=T_{n}(f)-H_{n}(f),
$$

where $H_{n}(f)$ is the centrosymmetric Hankel matrix generated by $f$. A Hankel matrix is such that its entries are constant along any lower-left-upper-right diagonal: with the same notations we have

$$
H_{n}(f)=\sum_{2 e \leqslant j \leqslant n-e} a_{j} K_{n}^{[j]}=\sum_{2 \leqslant\left|j_{1}\right| \leqslant n_{1}-1} \cdots \sum_{2 \leqslant\left|j_{d}\right| \leqslant n_{d}-1} a_{\left(j_{1}, \ldots, j_{d}\right)} K_{n_{1}}^{\left[j_{1}\right]} \otimes \cdots \otimes K_{n_{d}}^{\left[j_{d}\right]},
$$

where, in the unilevel case, $K_{n}^{[j]}$ denotes the matrix of order $n$ whose entry $(s, t)$ equals 1 if $s+t=j \bmod 2(n-1)$ and equals zero otherwise: the multilevel version of $K_{n}^{[j]}$ is now defined via (1.3).

A third class of matrices which form an algebra and is of interest in applications is represented by the Hartley matrices [4]. Unlike circulants and $\tau$ matrices, the Hartley class does not have a generator, but it can be described by using circulant matrices. In actuality, every matrix belonging to this class can be expressed as the sum of two independent matrices, the first being symmetric and circulant, the second being the product of a special permutation matrix $J$ by a skewcirculant matrix. More precisely, for a Hartley matrix generated by a unilevel function $f$ we set $J_{1,1}=J_{s, n+2-s}=1$, $s=2, \ldots, n$, and

$$
\mathcal{H}_{n}(f)=\mathcal{C}_{n}\left(f_{\text {even }}\right)+J \mathcal{C}_{n}\left(f_{\text {odd }}\right)
$$


where $f_{\text {even }}(x)=(f(x)+f(-x)) / 2$ and $f_{\text {odd }}(x)=(f(x)-f(-x)) / 2$. In this way the first column of $\mathcal{C}_{n}\left(f_{\text {even }}\right)$ has $\alpha_{j}$ coefficients such that $\alpha_{j}=\alpha_{n-j} \in \mathbb{R}, j=1, \ldots, n-1$, and the first column of $\mathcal{C}_{n}\left(f_{\text {odd }}\right)$ has coefficients $\beta_{j}=-\beta_{n-j} \in \mathbb{R}, j=1, \ldots, n-1$, $\beta_{0}=0$, where $\alpha_{0}=a_{0}$ and $\left(\alpha_{j}-\mathbf{i} \beta_{j}\right) / 2=a_{j}$ for $|j| \geqslant 1$. We note that its multilevel version amounts to performing the same even/odd splitting of $f$ with respect to each variable separately.

Since circulants, $\tau$ and Hartley matrices are algebras, they are all simultaneously diagonalized by a given transform. In our case the involved transforms are all unitary (or real unitary, i.e., orthogonal) and therefore these algebras are constituted by normal matrices. More precisely the three classes can be formally defined as follows:

$$
\mathcal{G}\left(Q_{n}\right)=\left\{Q_{n} \cdot \operatorname{Diag}(\mathbf{d}) \cdot Q_{n}^{-1} \mid \mathbf{d} \in \mathbb{C}^{n}\right\}=\left\{Q_{n} \cdot \operatorname{Diag}(\mathbf{d}) \cdot Q_{n}^{H} \mid \mathbf{d} \in \mathbb{C}^{n}\right\},
$$

where the related transforms $Q_{n}$ (and some other information, such as the grid points $w_{i}^{[n]}$, the $\mathcal{I}_{n}$ index range to which $i$ belongs, and the name of the class $\mathcal{C}, \tau, \mathcal{H}$ generically denoted by $\mathcal{A}$ ) are listed in the subsequent Table 1.1.

TABLE 1.1

Basics on our algebras: the unilevel case.

\begin{tabular}{|l||c|c|c|c|}
\hline & $\mathcal{A}$ & $\mathcal{I}_{n}$ & $\mathbf{w}^{[n]}$ & $Q_{n}$ \\
\hline \hline Circulants & $\mathcal{C}$ & $0, \ldots, n-1$ & $w_{i}^{[n]}=\frac{2 \pi i}{n}$ & $F_{n}=\frac{1}{\sqrt{n}}\left[e^{\mathbf{i} j w_{i}^{[n]}}\right]_{i, j=0}^{n-1}$ \\
\hline Hartley & $\mathcal{H}$ & $0, \ldots, n-1$ & $w_{i}^{[n]}=\frac{2 \pi i}{n}$ & $\operatorname{Re}\left(F_{n}\right)+\operatorname{Im}\left(F_{n}\right)$ \\
\hline Tau & $\tau$ & $1, \ldots, n$ & $w_{i}^{[n]}=\frac{\pi i}{n+1}$ & $\sqrt{\frac{2}{n+1}}\left[\sin \left(j w_{i}^{[n]}\right)\right]_{i, j=1}^{n}$ \\
\hline
\end{tabular}

Once again, whenever $n$ is a $d$-index we define $Q_{n}$ the matrix of size $N(n)$ as $Q_{n_{1}} \otimes$ $\cdots \otimes Q_{n_{d}}$. The matrices $\mathcal{C}_{n}(f), \tau_{n}(f)$, and $\mathcal{H}_{n}(f)$ can be written (in order to provide a uniform approach) as

$$
\mathcal{A}_{n}(f)=Q_{n} \cdot \operatorname{Diag}\left(f\left(\mathbf{w}^{[n]}\right)\right) \cdot Q_{n}^{H},
$$

where $\mathcal{A} \in\{\mathcal{C}, \mathcal{H}, \tau\}, f$ is a polynomial of degree less than $n$, and the vectors $\mathbf{w}^{[n]}$ are defined in the fourth column of Table 1.1 for scalar $n$ and $\mathbf{w}^{[n]}=\mathbf{w}^{\left[n_{1}\right]} \times \cdots \times \mathbf{w}^{\left[n_{d}\right]}$ if $n$ is a $d$-index. For instance, in the circulant case we observe $Q_{n}=F_{n}$ and we write $\mathcal{A}_{n}(f)=\mathcal{C}_{n}(f)=F_{n} \cdot \operatorname{Diag}\left(f\left(\mathbf{w}^{[n]}\right)\right) \cdot F_{n}^{H}$.

It is immediate to see that $\mathcal{C}_{n}(f), \tau_{n}(f)$, and $\mathcal{H}_{n}(f)$ are definitely ill-conditioned if $f$ has zeros in its basic definition set $[-\pi, \pi]^{d}$ (they are singular if the zeros contain a grid point). It is interesting to recall that $x=0$ is always a grid point for the circulants and the Hartley matrices so that $\mathcal{C}_{n}(f)$ and $\mathcal{H}_{n}(f)$ are singular if these matrices arises from the discretization of constant coefficients differential operators: in that case it is known that $x=0$ is a zero of the symbol and its order is associated to the maximal order of the involved derivatives (see, e.g., [30]).

In such a case, setting $\mathbf{e}=\sum_{j=1}^{N(n)} \mathbf{e}_{j}$, the classical Strang circulant preconditioner (see, e.g., [9]) is replaced by its modified (or stabilized) version (see, e.g., [34]):

$$
\widetilde{\mathcal{C}}_{n}(f)=\mathcal{C}_{n}(f)+\left(\min _{\|j\|_{\infty}=1} f\left(\mathbf{w}_{j}^{[n]}\right)\right) \frac{\mathbf{e e}^{t}}{N(n)} .
$$


Of course the same approach can be followed in the case of the Hartley algebra.

In this paper we are interested in the solution of linear systems with matrices of the form $\mathcal{A}_{n}(f)$ for $\mathcal{A} \in\{\mathcal{C}, \mathcal{H}, \tau, T\}$ and $f$ trigonometric polynomial. More specifically we are interested in (iterative) methods that show the best possible asymptotic complexity. In this respect we define a formal notion of optimality of an iterative method for a sequence of linear systems of increasing dimensions.

DeFinition 1.1. Given a sequence of linear systems of increasing dimensions $\left\{A_{n} \mathbf{x}_{n}=\mathbf{b}_{n}\right\}$, we write that an iterative method is optimal if

1. the arithmetic cost of every iteration is at most proportional to the complexity of a matrix vector product with matrix $A_{n}$,

2. the number of iterations for reaching the solution within a fixed accuracy can be bounded from above by a constant independent of $n$.

Such a method would be interesting in the case of the considered matrix algebras $\mathcal{A} \in\{\mathcal{C}, \mathcal{H}, \tau\}$ since the cost by direct methods using fast transforms is $O(N(n) \log N(n))$ while an optimal technique would require just $O(N(n))$ operations: we recall that this kind of matrix algebra linear systems are widely used as preconditioners for more complicated problems (dense Toeplitz, differential problems discretizations etc. $[13,25,30]$ ) or directly arise in the discretization of image restoration problems with shift-invariant kernel and suitable boundary conditions (see [20, 29]).

In the case of Toeplitz systems the improvement would be much more striking. For instance, in the multilevel Toeplitz setting the fast direct techniques are expensive because they are unable to exploit the Toeplitzness at each level. Concerning the preconditioned conjugate gradient (PCG) method, the matrix algebra preconditioners lead to optimal solvers only in the unilevel case (see, e.g., [9]). Unfortunately, for multilevel problems the optimal preconditioning by matrix algebras is simply impossible in general as proved by the last author and Tyrtyshnikov [32] (see also [21, 22, 26, 33]). More precisely the number of iterations is an unbounded function as $n$ and it is of the order of $O\left([N(n)]^{\frac{d-1}{d}}\right)$, which is very unsatisfactory if $d$ is large.

On the other hand, by using band Toeplitz preconditioners (see, e.g., [13]), it is possible to reduce the computation with dense Toeplitz systems to the case of Toeplitz linear systems whose coefficient matrices are generated by nonnegative polynomials. Therefore it is of special interest to be able to solve in optimal time (i.e., computational effort linear with respect to the size of the algebraic problem) linear systems whose coefficient matrix is of the form $T_{n}(f)$ with nonnegative polynomial $f$ and our proposal is the multigrid technique. We will give a formal proof of optimality of the $\mathrm{V}$-cycle multigrid iteration (MGM) in the matrix algebra case while in the Toeplitz case this optimal behavior is demonstrated only by numerical experiments (for a formal proof of optimal convergence rate, i.e., independent of $n$, related to the two-grid method refer to [27]): our hope is that the theoretical tools introduced in this paper for the matrix algebra case could be used for proving the V-cycle optimality in the Toeplitz context as well. We stress that the proof technique introduced in this paper seems to be new compared with the classical approaches used in the PDEs context (see, e.g., the beautiful review [37]). Indeed our tools are totally matrix oriented so that there is no differential interpretation in the general case: for instance when the symbol has a zero close to $\pi$, then the smoother (i.e., the iteration satisfying the smoothing property according to Ruge and Stüben [23]) does not make the error smooth; i.e., it reduces the components in the low frequencies and it does not reduce the components in the high frequencies.

Finally, we mention that our technique can be easily extended with the same 
linear arithmetic cost to linear systems with coefficient matrices given by $\mathcal{A}_{n}(f)+$ $\sum_{t=1}^{s} \vartheta_{t} \cdot q_{i_{t}}^{[n]}\left(q_{i_{t}}^{[n]}\right)^{H}=\mathcal{A}_{n}\left(f+\sum_{t=1}^{s} \vartheta_{t} \chi_{w_{i_{t}}^{[n]}+2 \pi \mathbb{Z}}\right)$, where the vector $q_{i}^{[n]}$ is the $i$ th column of $Q_{n}, \mathcal{A} \in\{\mathcal{C}, \mathcal{H}, \tau\}$, and $\chi_{S}$ denotes the characteristic function of a given set $S$. We recall that these examples of coefficient matrices are a generalization of the stabilized Strang preconditioner displayed in (1.5).

The paper is organized as follows. In section 2 we first introduce the multigrid procedure by reporting the basic convergence results by Ruge and Stüben [23]; then we describe our choices for the smoothing and prolongation operators. In section 3 we show that "level independency" property is not sufficient to reach the optimality, while in section 4 we prove the optimal convergence rate of our multigrid in the unilevel case. Section 5 is devoted to the multilevel case, while in section 6 we generalize our V-cycle algorithm to multilevel Toeplitz matrices. Section 7 contains wide numerical experimentation that confirms the theoretical analysis, and section 8 is devoted to concluding remarks, open problems and future work.

2. The multigrid procedure. Let $A \in \mathbb{C}^{n \times n}$ be a Hermitian positive definite matrix, $\mathbf{b} \in \mathbb{C}^{n}, m$ integer with $0<m<n$. Fix integers $n_{0}=n>n_{1}>n_{2}>\cdots>$ $n_{m}>0$, take $P_{i+1}^{i} \in \mathbb{C}^{n_{i+1} \times n_{i}}$ full-rank matrices, and consider a class $\mathcal{R}_{i}$ of iterative methods for $n_{i}$-dimensional linear systems. The related V-cycle method (see [5, 17]) produces the sequence $\left\{\mathbf{x}^{(k)}\right\}_{k \in \mathbb{N}}$ according to the rule $\mathbf{x}^{(k+1)}=\mathcal{M G M}\left(0, \mathbf{x}^{(k)}, \mathbf{b}\right)$, with $\mathcal{M G M}$ recursively defined as follows:

$$
\begin{aligned}
& \mathbf{x}_{i}^{\text {(out) }}:=\mathcal{M G M}\left(i, \mathbf{x}_{i}^{(\text {in })}, \mathbf{b}_{i}\right) \\
& \text { If }(i=m) \text { Then Solve }\left(A_{m} \mathbf{x}_{m}^{\text {(out) }}=\mathbf{b}_{m}\right) \\
& \text { Else } \mathbf{1} \mid \mathbf{r}_{i} \quad:=A_{i} \mathbf{x}_{i}^{(\text {in })}-\mathbf{b}_{i} \\
& 2 \quad \mathbf{b}_{i+1}:=P_{i+1}^{i} \mathbf{r}_{i} \\
& 3 \quad A_{i+1}:=P_{i+1}^{i} A_{i}\left(P_{i+1}^{i}\right)^{H} \\
& 4 \mathbf{y}_{i+1}:=\mathcal{M G M}\left(i+1, \mathbf{0}_{n_{i+1}}, \mathbf{b}_{i+1}\right) \\
& 5 \quad \mathbf{x}_{i}^{\text {(int) }}:=\mathbf{x}_{i}^{\text {(in) }}-\left(P_{i+1}^{i}\right)^{H} \mathbf{y}_{i+1} \\
& 6 \mathbf{x}_{i}^{\text {(out) }}:=\mathcal{R}_{i}^{\nu}\left(\mathbf{x}_{i}^{(\text {int })}\right) .
\end{aligned}
$$

Step 1 calculates the residual of the proposed solution; steps 2, 3, 4, and 5 define the recursive coarse grid correction by projection (2) of the residual, sub-grid correction $(3,4)$ and interpolation (5), while step 6 performs some $(\nu)$ iterations of a "postsmoother."

By using the MGM as an iterative technique, at the $k$ th iteration, we obtain the linear systems $A_{i} \mathbf{x}_{i}^{(k)}=\mathbf{b}_{i}^{(k)}, i=0, \ldots, m$, where the matrices $A_{i} \in \mathbb{C}^{n_{i} \times n_{i}}$ are Hermitian positive definite. Only the last is solved exactly while all the others are recursively managed by reduction to low-level system and smoothing. $\mathcal{R}_{i}$ are most of the time one-point methods (see [23]) with prescribed linear part $R_{i} \in \mathbb{C}^{n_{i} \times n_{i}}$ i.e.,

$$
\mathcal{R}_{i}\left(\mathbf{x}_{i}\right)=R_{i} \mathbf{x}_{i}+\left(I_{n_{i}}-R_{i}\right) A_{i}^{-1} \mathbf{b}_{i}^{(k)}, \quad \mathbf{x}_{i} \in \mathbb{C}^{n_{i}}, \quad i=0, \ldots, m-1 .
$$

If we define the multigrid iteration matrix of level $i$ as $M G M_{i}$,

$$
\left\{\begin{array}{l}
M G M_{m}=O_{n_{m} \times n_{m}}, \\
M G M_{i}=R_{i}^{\nu} \cdot\left[I_{n_{i}}-\left(P_{i+1}^{i}\right)^{H}\left(I_{n_{i+1}}-M G M_{i+1}\right) A_{i+1}^{-1} P_{i+1}^{i} A_{i}\right], \quad i=m-1, \ldots, 0,
\end{array}\right.
$$


it holds that $\mathbf{x}_{i}^{\text {(out) }}=M G M_{i} \mathbf{x}_{i}^{\text {(in) }}+\left(I_{n_{i}}-M G M_{i}\right) A_{i}^{-1} \mathbf{b}_{i}$, so in the finer grid we have $\mathbf{x}^{(\mathrm{k}+1)}=M G M_{0} \mathbf{x}^{(\mathrm{k})}+\left(I_{n_{0}}-M G M_{0}\right) A_{i}^{-1} \mathbf{b}$, and $M G M_{i}$ depends on $i$ but not on any $\mathbf{x}_{i}^{(k)}$ nor on $\mathbf{b}_{i}^{(k)}$. The algorithm has essentially two degrees of indetermination:

1. choice of the projectors $P_{i+1}^{i}, i=0, \ldots, m-1$;

2. choice of the smoothers $\mathcal{R}_{i}, i=0, \ldots, m-1$.

The choice of the projectors $P_{i+1}^{i}$ and the calculation of the matrices $A_{i}$ are performed before the beginning of the $\mathrm{V}$-cycle procedure (precomputing phase).

Of course $m$ stands for the number of subgrids in the algorithm. We also refer the choice $m=1$ as two-grid method (TGM), so we define the TGM linear action as

$$
T G M_{0}=R_{0}^{\nu} \cdot\left[I_{n_{0}}-\left(P_{1}^{0}\right)^{H} A_{1}^{-1} P_{1}^{0} A_{0}\right] .
$$

The term in square brackets in (2.4) is defined as exact coarse grid correction $\left(C G C_{0}\right)$. It can be defined of course on each grid of the $\mathrm{V}$-cycle algorithm, and hence we agree to write

$$
C G C_{i}=I_{n_{i}}-\left(P_{i+1}^{i}\right)^{H} A_{i+1}^{-1} P_{i+1}^{i} A_{i}, \quad i=0, \ldots, m-1,
$$

and

$$
T G M_{i}=R_{i}^{\nu} \cdot C G C_{i}, \quad i=0, \ldots, m-1 .
$$

Specific TGMs and V-cycles have been devised for the $\tau$ multilevel algebra $[15,16]$, while for multilevel circulants they have been studied in [31].

2.1. Convergence related theorems. Here we recall two theorems [23] concerning the convergence of multigrid iterations. The first one is related to the easier TGM algorithm; the other refers to the complete (i.e., with $m>1$ ) multigrid procedure. For the sake of simplicity, in both the theorems we will assume just one application of the smoother, i.e., $\nu=1$. By $\|\cdot\|_{2}$ we denote the Euclidean norm on $\mathbb{C}^{n}$ and the associated induced norm on $\mathbb{C}^{n \times n}$; if $X$ is positive definite we also denote $\|\cdot\|_{X}=\left\|X^{1 / 2} \cdot\right\|_{2}$, and whenever $X$ and $Y$ are both Hermitian matrices the notation $X \geqslant Y$ means that $X-Y$ is positive semidefinite.

TheOREM 2.1 (TGM convergence [23]). Let $n_{0}, n_{1}$ be integers such that $n_{0}>$ $n_{1}>0$ and let $A \in \mathbb{C}^{n_{0} \times n_{0}}$ be a positive definite Hermitian matrix, $\mathbf{b} \in \mathbb{C}^{n_{0}}$, and also let $\mathcal{R}_{0}$ be defined as in (2.2). Fix $P_{1}^{0} \in \mathbb{C}^{n_{1} \times n_{0}}$ full-rank matrix and let $D=$ $\operatorname{Diag}\left[a_{i i}\right]_{i=1}^{n_{0}}$ be the main diagonal of $A$. Suppose that $\alpha>0$ exists such that

$$
\left\|R_{0} \mathbf{x}\right\|_{A}^{2} \leqslant\|\mathbf{x}\|_{A}^{2}-\alpha\|\mathbf{x}\|_{A D^{-1} A}^{2} \quad \forall \mathbf{x} \in \mathbb{C}^{n_{0}} .
$$

Then for each $\gamma>0$ such that

$$
\min _{\mathbf{y} \in \mathbb{C}^{n_{1}}}\left\|\mathbf{x}-\left(P_{1}^{0}\right)^{H} \mathbf{y}\right\|_{D}^{2} \leqslant \gamma\|\mathbf{x}\|_{A}^{2} \quad \forall \mathbf{x} \in \mathbb{C}^{n_{0}}
$$

it holds that $\alpha \leqslant \gamma$ and

$$
\left\|T G M_{0}\right\|_{A} \leqslant \sqrt{1-\alpha / \gamma}<1 .
$$

From Theorem 2.1, it follows that $\left\{\mathbf{x}^{(k)}\right\}_{k}$ converges to the solution of $A \mathbf{x}=\mathbf{b}$. Furthermore, when $\alpha$ and $\gamma$ are independent of $n$, the sequence $\left\{\mathbf{x}^{(k)}\right\}_{k}$ converges with (at least) a constant error reduction by the factor $\sqrt{1-\alpha / \gamma}$ independent of the 
dimension $n$ of the system: therefore the corresponding TGM has optimal convergence rate (i.e., it satisfies the second item in Definition 1.1).

Remark 2.2. Theorem 2.1 still holds if $D \in \mathbb{C}^{n_{0} \times n_{0}}$ is replaced by any Hermitian positive definite matrix $X$ ( $X=I_{n_{0}}$ could be a suitable choice): it is enough to repeat verbatim the proof of Theorem 5.2 in [23] with $X$ in place of $D$.

TheOREM 2.3 (MGM convergence [23]). Let $m, n$ be integers satisfying $0<m<$ $n$ and suppose that $A \in \mathbb{C}^{n \times n}$ is a positive definite Hermitian matrix and $\mathbf{b} \in \mathbb{C}^{n}$; given now a sequence of $m+1$ positive integers $n=n_{0}>n_{1}>\cdots>n_{m}$, let $P_{i+1}^{i} \in \mathbb{C}^{n_{i+1} \times n_{i}}$ be full-rank matrices for each $i=0, \ldots, m-1$. Define $A_{0}=A$ and choose a class of iterative methods $\mathcal{R}_{i}$ as in (2.2). If there exists a real positive number $\delta$ satisfying

$$
\left\|R_{i} \mathbf{x}\right\|_{A_{i}}^{2} \leqslant\|\mathbf{x}\|_{A_{i}}^{2}-\delta\left\|C G C_{i} \mathbf{x}\right\|_{A_{i}}^{2} \quad \forall \mathbf{x} \in \mathbb{C}^{n_{i}}
$$

for every $i=0, \ldots, m-1$, then it holds $\delta \leqslant 1$ and

$$
\left\|M G M_{0}\right\|_{A} \leqslant \sqrt{1-\delta}<1 .
$$

As in the case of the TGM, here also the sequence $\left\{\mathbf{x}^{(k)}\right\}_{k}$ converges to the solution of $A \mathbf{x}=\mathbf{b}$ and when $\delta$ is independent of $n$ it converges with at least a constant error reduction not depending on the dimension of the system and, at most, $\left\lceil 2 \delta^{-1} \ln \left(\varepsilon^{-1}\right)\right\rceil$ iterations are needed to reduce the error by a factor $\varepsilon>0$.

We observe that inequality (2.9) is easily guaranteed by the following:

$$
\begin{aligned}
\left\|R_{i} \mathbf{x}\right\|_{A_{i}}^{2} \leqslant\|\mathbf{x}\|_{A_{i}}^{2}-\alpha_{i}\|\mathbf{x}\|_{A_{i}^{2}}^{2} & \left(\alpha_{i}>0\right) & \forall \mathbf{x} \in \mathbb{C}^{n_{i}} \\
\left\|C G C_{i} \mathbf{x}\right\|_{A_{i}}^{2} \leqslant \beta_{i}\|\mathbf{x}\|_{A_{i}^{2}}^{2} & & \forall \mathbf{x} \in \mathbb{C}^{n_{i}} .
\end{aligned}
$$

If $\delta \leqslant \alpha_{i} / \beta_{i}$, then (2.9) holds for every $i=0, \ldots, m-1$ with the choice of $\delta=$ $\min _{0 \leqslant i \leqslant m-1}\left\{\alpha_{i} / \beta_{i}\right\}$. We refer to (2.11a) as the smoothing property and to (2.11b) as the approximation property (see [23, 37]). The approximation property depends exclusively on the choice of projectors (i.e., $P_{i+1}^{i}$ ) but not on smoothers, whereas smoothing property is not related to $P_{i+1}^{i}$. The separate study of these two properties allows us to cope with the difficult part of the procedure (the verification of condition (2.11b)) involving the projectors but not depending on the smoothers. Notice that the direct verification of (2.9) is in principle much more intricate due to the simultaneous presence of the projectors and of the smoothers in the inequalities.

Remark 2.4. The MGM smoothing property (2.11a) is nothing more than the TGM smoothing property (2.7a) with $D$ substituted by $I$, in accordance with Remark 2.2 .

In such a situation, optimality is reached if $\delta$ is independent from both $n$ and $m$, i.e., it suffices to show that a constant value $\delta$ exists such that $0<\delta \leqslant \min _{i}\left\{\alpha_{i} / \beta_{i}\right\}$ is fulfilled for every possible choice of $n$ and $m$. In this way, the number of iterations required keeps being uniformly bounded by a constant irrespective of the dimension of the problem. What is more, since each iteration has a computational cost proportional to matrix-vector product, Definition 1.1 states that such a kind of MGM is optimal.

2.2. MGM for matrix algebras. We analyze a special instance of the MGM (2.1), introduced in $[15,16,31]$, where the smoother is the relaxed Richardson iteration, namely $R_{i}=I_{n_{i}}-\omega_{i} A_{i}$ ( $\omega_{i}$ is relaxing parameter), and on each step we essentially halve the dimension $\left(n_{i+1}=\frac{n_{i}}{2}\right.$ for circulants and Hartley and $n_{i+1}=\frac{n_{i}-1}{2}$ for $\tau$ matrices). 
Dealing with circulants or Hartley matrices we start from dimension $n_{0}=2^{k_{0}}$ and define the subgrid dimensions as $n_{i}=2^{k_{0}-i}$, while in $\tau$ algebra we start with $n_{0}=2^{k_{0}}-1$ and define $n_{i}=2^{k_{0}-i}-1$. The cutting operator is defined by $K_{i+1}^{i}$ : $\mathbb{C}^{n_{i}} \longrightarrow \mathbb{C}^{n_{i+1}}$ and it selects even index components (we recall that the index range is $\left\{0, \ldots, n_{i}-1\right\}$ in the circulant and Hartley algebras, while it is $\left\{1, \ldots, n_{i}\right\}$ in the $\tau$ algebra):

Circulant \& Hartley algebra $\quad \tau$ algebra

$$
\begin{aligned}
& n_{i}=2^{k_{0}-i} \\
& n_{i}=2^{k_{0}-i}-1
\end{aligned}
$$

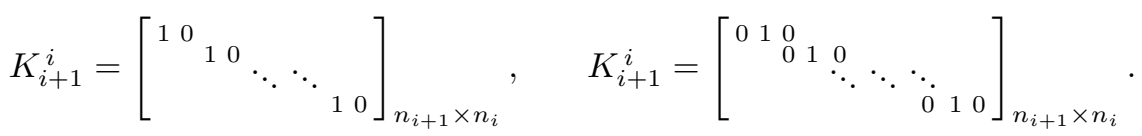

We have defined the projector in the form $P_{i+1}^{i}=K_{i+1}^{i} \cdot \mathcal{A}_{n_{i}}\left(p_{i}\right)$ while $p_{i}$ is a real valued polynomial which will be chosen in section 4.2 in order to satisfy the approximation property (2.11b). Our choices on $K_{i+1}^{i}$ brings (see [31]) to $K_{i+1}^{i} Q_{n_{i}}=\left[Q_{n_{i+1}} \mid Q_{n_{i+1}}\right]$ in the case of the circulant and Hartley algebras, while dealing with $\tau$ matrices we have $K_{i+1}^{i} Q_{n_{i}}=\left[Q_{n_{i+1}}\left|\mathbf{0}_{n_{i}+1}\right|-J_{n_{i+1}} Q_{n_{i+1}}\right]$ with $\left[J_{n}\right]_{h, k}$ equals 1 if $h+k=n+1$ and 0 if not. These two equalities play a basic role in maintaining the matrix algebra structure on subgrids and represent the keystone for proving the following proposition.

Proposition 2.5 (see $[27,31]$ ). Let $k_{0}, m$ be integers such that $0<m<k_{0}$, $f_{0}$ and $p_{i}, i=0, \ldots, m-1$, be real $2 \pi$-periodic functions (also even in the $\tau$ case), $P_{i+1}^{i}=K_{i+1}^{i} \cdot \mathcal{A}_{n_{i}}\left(p_{i}\right)$ with $\mathcal{A} \in\{\mathcal{C}, \mathcal{H}, \tau\}$ as in (1.4). Also define $A_{0}=\mathcal{A}_{n_{0}}\left(f_{0}\right)$ and $A_{i+1}=P_{i+1}^{i} A_{i}\left(P_{i+1}^{i}\right)^{H}$ for $i=0, \ldots, m-1$. Then it holds that $A_{i+1}=\mathcal{A}_{n_{i+1}}\left(f_{i+1}\right)$, where

$$
f_{i+1}(x)=\frac{1}{2}\left[\left(p_{i}^{2} f_{i}\right)\left(\frac{x}{2}\right)+\left(p_{i}^{2} f_{i}\right)\left(\pi+\frac{x}{2}\right)\right], \quad i=0, \ldots, m-1 .
$$

Moreover each projector $P_{i+1}^{i}$ is full-rank if $p_{i}^{2}(x)+p_{i}^{2}(\pi+x)>0$ holds true for every $x$.

Proposition 2.5 is basic for our purposes because it allows one to relate the functions $f_{i}$ to the matrices $A_{i}$ in the V-cycle procedure (2.1). Furthermore, we observe that

$$
h(x)=\sum_{j=k_{1}}^{k_{2}} a_{j} e^{\mathbf{i} j x} \quad \Rightarrow \quad h\left(\frac{x}{2}\right)+h\left(\pi+\frac{x}{2}\right)=2 \sum_{j=\left\lceil\frac{k_{1}}{2}\right\rceil}^{\left\lfloor\frac{k_{2}}{2}\right\rfloor} a_{2 j} e^{\mathbf{i} j x}
$$

represents a fundamental simplification in checking convergence and in evaluating the computational costs. By defining $\mathbb{R}_{k}[x]=\left\{\sum_{|j| \leqslant k} a_{j} e^{\mathbf{i} j x} \mid a_{j}=\bar{a}_{-j} \in \mathbb{C}\right\}$, and by assuming $f(x) \in \mathbb{R}_{T_{0}}[x], p_{i}(x) \in \mathbb{R}_{q_{i}}[x]$, we have $f_{i} \in \mathbb{R}_{T_{i}}$ with $T_{i+1}=q_{i}+\left\lfloor\frac{T_{i}}{2}\right\rfloor$, and, by induction, we deduce $T_{i} \leqslant \max \left\{T_{0} ; 2 q_{j}-1: 1 \leqslant j \leqslant i\right\}$. Consequently the bandwidth of $\mathcal{A}_{i}$ is uniformly bounded if there exists a constant $T$ such that $T_{0}, q_{i} \leqslant T$ for every $i$. Furthermore, if $q_{i}=q$ holds for every $i$, then $T_{i} \uparrow 2 q-1$ (monotonic nondecreasing convergence) if $T_{0} \leqslant 2 q-1, T_{i} \downarrow 2 q$ (monotonic nonincreasing convergence) otherwise.

The subsequent theorem has been proven in $[27,31]$, where it has been used for proving the TGM optimality if a good choice of $p_{0}$ is performed. 
Theorem 2.6 (see $[27,31]$ ). Let $A_{n_{0}}=\mathcal{A}_{n}\left(f_{0}\right)$ with $\mathcal{A} \in\{\mathcal{C}, \mathcal{H}, \tau\}$, $f_{0}$ be nonnegative, $2 \pi$-periodic (even in the $\tau$ case), and let $P_{1}^{0}=K_{1}^{0} \cdot \mathcal{A}_{n_{0}}\left(p_{0}\right)$, with $p_{0}$ trigonometric polynomial (also even in $\tau$ case) such that $f_{0}\left(x^{0}\right)=0$ implies

$$
\begin{aligned}
\lim _{x \rightarrow x^{0}} \frac{p_{0}^{2}(\pi+x)}{f_{0}(x)}<+\infty, & \\
p_{0}^{2}(x)+p_{0}^{2}(\pi+x) & >\quad 0 \quad \forall x .
\end{aligned}
$$

Then inequality (2.7b) in Theorem 2.1 is satisfied.

In what follows we will need a stronger version of $(2.15 \mathrm{a})$ to prove the $\mathrm{V}$-cycle optimality.

3. Level independency does not imply multigrid optimality. An informal but dangerous (as we will see) way of defining the MGM is as recursive application of TGM iterations. In particular, if the convergence rate $\sqrt{1-\alpha / \gamma}$ defined in (2.8) is independent of the recursion level, we have a property known in literature as "level independency" [7].

DeFINITION 3.1. Let $m, n$ be integers satisfying $0<m<n$ and let us suppose that we solve a system of dimension $n$ with MGM. Then we have level independency if the method TGMi induced on each level satisfies

$$
\left\|T G M_{i}\right\|_{A_{i}} \leqslant c<1, \quad i=0, \ldots, m-1,
$$

with $c$ pure constant independent of $n$ and $m$.

In some recent works, the level independency was indicated as a way for obtaining the V-cycle optimality (see, e.g., [7]). Actually, we will prove that the level independency is necessary but not sufficient for the MGM optimality. To explain this fact intuitively, we observe that to consider the MGM as a recursive TGM application is equivalent to having the exact knowledge of the error at each level, since the TGM directly solves the system at the lower level. Indeed, for applying the TGM recursively, we must only decide if the recursive call should be placed before or after the direct resolution of the lower level system. It follows that in the first case we project the problem at the lower level as for the MGM, but when we interpolate the solution (the error) at each level this is exactly known at the lower level and it does not derive from previous interpolation as for the MGM. In the second case we know exactly the error that we project at each level, while for the MGM this derives from previous projections. On the other side, MGM replaces the direct solution of the system with the recursive call, obtaining a more approximate procedure with respect to the recursive TGM application. Therefore, the level independency is a necessary but not sufficient condition for the MGM optimality.

Now we report a whole class of counterexamples to enhance the previous informal description.

Proposition 3.2. Let $A=\tau_{n_{0}}\left(f_{0}\right), P_{i+1}^{i}=K_{i+1}^{i} \tau_{n_{i}}(p)$, and

$$
\begin{aligned}
f_{0}(x) & =(1-\cos (x))^{q}, & & q \in \mathbb{N}, \\
p(x) & =\mu^{\left\lceil\frac{q}{2}\right\rceil}(1+\cos (x))^{\left\lceil\frac{q}{2}\right\rceil}, & & \mu \in \mathbb{R}, \mu \neq 0,
\end{aligned}
$$

for $i=0, \ldots, m-1$. Then the level independency property holds for the MGM applied to the system $A \mathbf{x}=\mathbf{b}, \mathbf{x}, \mathbf{b} \in \mathbb{C}^{n_{0}}$, where $P_{i+1}^{i}$ is the projector at the level $i$.

Proof. Following Definition 3.1 we must prove that $\left\|T G M_{i}\right\|_{A_{i}} \leqslant c<1$ with $c$ absolute constant and, to this purpose, it is enough to prove $\theta_{i}=\sqrt{1-\alpha_{i} / \gamma_{i}} \leqslant c<1$ 
with $c$ constant and independent of $n$ for every level $i=0, \ldots, m-1$. At the moment we consider for simplicity only $\mu= \pm 2$, but at the end we will show that we can extend the proof for every nonzero $\mu \in \mathbb{R}$. At the first level with $f_{0}$ and $p_{0}=p$ we are in the hypotheses of Theorem 2.6, therefore, at the first level, the TGM converges with convergence rate $\theta_{0}<1$. Using the function relation (2.13) to find $f_{i+1}$ from $f_{i}$ and $p_{i}$, we have to distinguish between $q$ even and odd.

- $q$ even: In this case we obtain $f_{1}=f_{0}$ so that $p_{1}=p_{0}$ satisfies again the conditions $(2.15)$ and then $\theta_{1}=\theta_{0}$. Iteratively $f_{i+1}=f_{i}=\cdots=f_{0}$ with $p_{i}=\cdots=p_{0}=p$ for $1 \leqslant i \leqslant m-1$ and $\theta_{i}=\theta_{0}$.

- $q$ odd: Here we have $f_{1}=2 f_{0}$, then $p_{1}=p_{0}$ satisfies again the conditions (2.15) and iteratively $f_{i+1}=2 f_{i}=\cdots=2^{i+1} f_{0}$ with $p_{i}=\cdots=p$ for $1 \leqslant i \leqslant m-1$. Even if $f_{i}$ changes at each level, in the computation of $\theta_{i}$ the factor $2^{i}$ is simplified out and then $\theta_{i}=\theta_{0}$ for every $i$.

If $\mu \neq \pm 2$, as in the case of $q$ odd, we obtain $f_{j}=\xi^{j} f_{0}, \xi \in \mathbb{R}$ and nonzero $\xi$, but again $\theta_{i}=\theta_{0}$.

In conclusion, with $c=\theta_{0}$ we have $\theta_{i}=c<1$ for every level $i=0, \ldots, m-1$, i.e., the level independency property is satisfied.

Remark 3.3. The previous proposition can be generalized to every function $f_{0}$ that vanishes at the origin with a zero of finite order. In particular, in this case, the level independency holds under the same TGM optimality conditions (2.15) and does not require more restrictive conditions.

Now we present an example where the projectors satisfy the previous proposition but are not sufficient for ensuring the V-cycle optimality. Moreover we will see that a slight modification of the proposed projectors will be enough for an optimal MGM convergence rate. We perform only a Richardson post-smoother iteration with $\omega=1 / \max \left(f_{0}\right)$ and MGM is stopped when $\left\|\mathbf{r}_{0}^{(k)}\right\|_{2} \leq 10^{-11}\|\mathbf{b}\|_{2}$. From the fourth derivative discretization by finite differences and appropriate boundary conditions, we obtain a system with coefficient matrix $T_{n}\left(f_{0}\right)$, where

$$
f_{0}(x)=(2-2 \cos (x))^{2} .
$$

We consider its $\tau$ version $\tau_{n_{0}}\left(f_{0}\right)$ (which corresponds to the natural $\tau$ preconditioner of $\left.T_{n_{0}}\left(f_{0}\right)\right)$. Therefore, defining the projector at each level through the trigonometric polynomial

$$
p(x)=2+2 \cos (x),
$$

we remark that the hypotheses of Proposition 3.2 are fulfilled and then the level independency property stands. From the numerical application of the corresponding V-cycle algorithm to the system $\tau_{n_{0}}\left(f_{0}\right) \mathbf{x}=\mathbf{b}$ with the proposed projector, we observe that the iteration number grows (almost linearly) as the dimension $n$ (refer to Table 3.1). Therefore, the proposed method is not optimally convergent while the level independency holds true. From the same table we can see that leaving unchanged the post-smoother and increasing the projector degree by 1 , it is possible to recover the MGM optimality. The last column in Table 3.1 stresses as the fundamental choice the projector and not the smoother, indeed, also increasing the Richardson iteration number and adding some conjugate gradient $(\mathrm{CG})$ iterations as post-smoother (accelerator), the MGM iteration number diverges as the problem dimension tends to infinity. We observe a similar behavior in image restoration problems: Compare these results with section 7.5 , especially Table 7.8 , and with $[6,18]$. 
TABLE 3.1

$M G M$ iteration number in the case of natural $\tau$ preconditioner for the monodimensional fourth derivative.

\begin{tabular}{|c|c|c|c|}
\hline \multirow[b]{2}{*}{$n$} & \multicolumn{2}{|c|}{1 Richardson iteration with $\omega=1 / \max \left(f_{0}\right)$} & \multirow{2}{*}{$\begin{array}{c}p(x)=2+2 \cos (x) \\
2 \text { Richardson with } \\
\omega=1 / \max \left(f_{0}\right) \text { and } \\
2 \text { CG iterations }\end{array}$} \\
\hline & $\begin{array}{c}p(x)=2+2 \cos (x) \\
\text { level independency holds but } \\
\text { the MGM is not optimal }\end{array}$ & $\begin{array}{c}p(x)=(2+2 \cos (x))^{2} \\
\text { MGM optimality and } \\
\text { level independency are satisfied }\end{array}$ & \\
\hline $2^{7}-1$ & 283 & 83 & 113 \\
\hline $2^{8}-1$ & 510 & 83 & 196 \\
\hline $2^{9}-1$ & 899 & 83 & 299 \\
\hline $2^{10}-1$ & 1541 & 83 & 475 \\
\hline
\end{tabular}

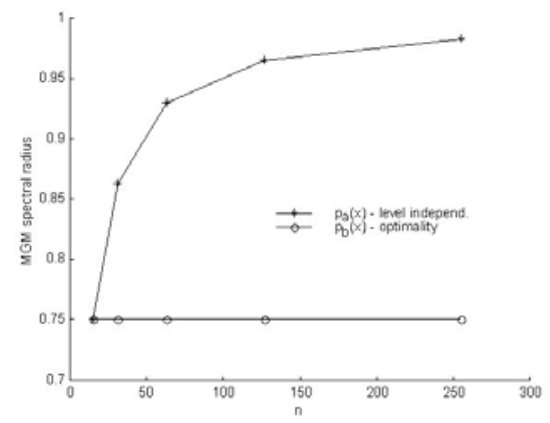

\begin{tabular}{|c|c|c|}
\hline \multirow{2}{*}{$\mathrm{n}$} & \multicolumn{2}{|c|}{$M G M_{0}$ spectral radius } \\
\cline { 2 - 3 } & $p_{a}(x)$ & $p_{b}(x)$ \\
\hline \hline 15 & 0.75 & 0.75 \\
31 & 0.8629 & 0.75 \\
63 & 0.9297 & 0.75 \\
127 & 0.9647 & 0.75 \\
255 & 0.9823 & 0.75 \\
511 & 0.9912 & 0.75 \\
\hline
\end{tabular}

FIG. 3.1. Spectral radius of $M G M_{0}$ with $p(x)=p_{a}(x)=2+2 \cos (x)$ (level independency but not optimality) and $p(x)=p_{b}(x)=(2+2 \cos (x))^{2}$ (level independency and optimality).

The difference between level independency and MGM optimality is underlined also from Figure 3.1, where it is shown the spectral radius of the MGM iteration matrix calculated by using recurrence (2.3). In our V-cycle algorithm, we solve the system at dimension 7 by a direct method. Therefore, at dimension 15 we have that the MGM is reduced to the TGM and we notice that the MGM with $p(x)=p_{a}(x)=2+2 \cos (x)$ has the same spectral radius as the MGM with $p(x)=p_{b}(x)=(2+2 \cos (x))^{2}$, due to the optimality of TGM.

It is starting from these remarks that in the next section we propose an optimal MGM and we prove its optimal behavior under mild assumptions on the symbol $f_{0}$.

4. Proof of convergence and optimality: The scalar case. We now show a way for satisfying the assumptions of Theorem 2.3, in their strong version (2.11a) and (2.11b). The first inequality (2.11a) is quite simple (i.e., polynomial) so it can be handled as in $[27,31]$. The second is more difficult to show and it represents one of the main contributions of the paper.

4.1. How to fulfill the smoothing property. We start with a result which is a slight variation of analogous propositions in $[27,31]$.

Proposition 4.1. For every $i=0, \ldots, m-1$, let $A_{i}=\mathcal{A}_{n_{i}}\left(f_{i}\right)$ with $\mathcal{A} \in\{\mathcal{C}, \mathcal{H}, \tau\}$ as in (1.4), $f_{i}$ being nonnegative, and let $\omega_{i}$ be such that $0<\omega_{i}<2 /\left\|f_{i}\right\|_{\infty}$. If we choose $\alpha_{i}$ fulfilling $\alpha_{i} \leqslant \omega_{i}\left(2-\omega_{i}\left\|f_{i}\right\|_{\infty}\right)$ and if we define $R_{i}=I_{n_{i}}-\omega_{i} A_{i}$, then

$$
\left\|R_{i} \mathbf{x}\right\|_{A_{i}}^{2} \leqslant\|\mathbf{x}\|_{A_{i}}^{2}-\alpha_{i}\|\mathbf{x}\|_{A_{i}^{2}}^{2}
$$

holds true for every $\mathbf{x} \in \mathbb{C}^{n}$. 
Proof. The essential steps for proving (4.1) can be found in [27, 31]. We just observe that the best bound to $\alpha_{i}$ is $1 /\left\|f_{i}\right\|_{\infty}$ and it is obtained by taking $\omega_{i}=\omega_{i}^{*}=$ $1 /\left\|f_{i}\right\|_{\infty}$.

4.2. How to fulfill the approximation property. We still have to show how to satisfy the more intricate MGM hypothesis (2.11b). We will consider the following set of conditions,

$$
p_{i}^{2}(x)+p_{i}^{2}(\pi+x)>0, \quad \limsup _{x \rightarrow x_{0}}\left|\frac{p_{i}(\pi+x)}{f_{i}(x)}\right|<+\infty, \quad i=0, \ldots, m-1,
$$

to hold for every $x$ and where $x_{0}$ is the unique zero of $f_{i}$ of order $2 q$. We observe that the conditions above are stronger than those (2.15) considered for the TGM method: a qualitative reasoning behind it is contained in section 3 and concerns the fact that the level independency does not imply the MGM optimality.

It follows that $p_{i}$ must possess a unique zero of the same (or higher) order as $f$. We will choose $p_{i}$ as follows:

$$
p_{x_{0}, q}^{\mathcal{C}, \mathcal{H}}(x)=\left[1+\cos \left(x-x_{0}\right)\right]^{q}, \quad p_{x_{0}, q}^{\tau}(x)=\left[\cos \left(x_{0}\right)+\cos (x)\right]^{q} \text { if } x_{0} \in\{0, \pi\} .
$$

In addition, if $f$ has a zero at $x_{0} \notin\{0, \pi\}$ and we are in the $\tau$ case, then $f$ is even and also has a zero at $2 \pi-x_{0}$ : in that case of two zeros we choose $p_{ \pm x_{0}, q}^{\tau}(x)=$ $\left[\cos \left(x_{0}\right)+\cos (x)\right]^{2 q}$. Finally, we consider a product of some of these basic polynomials in the general multiple-zeros case (see also [15, 27, 31]).

We will also use the following factorization result.

Proposition 4.2. Let $f$ be a trigonometric polynomial such that $f\left(x_{0}\right)=0$ and $f(x)>0$ whenever $x \not \equiv x_{0} \bmod 2 \pi$. Then there exists a positive trigonometric polynomial $\psi$ such that

$$
f(x)=\left[1-\cos \left(x-x_{0}\right)\right]^{q} \cdot \psi(x)
$$

and $2 q$ is the order of $f$ at $x_{0}$.

In the rest of the subsection and in section 4.3 we will focus our attention on the important case where the symbol has a unique zero at $x=0$ (this includes various discretized boundary values problems) with the exception of Proposition 4.5 and Remark 4.7: the more general case of a zero not at $x=0$ will be briefly treated in sections 4.4 and 4.5 .

Proposition 4.2 ensures a suitable factorization for our generating function $f_{0}$, i.e., $f_{0}(x)=[1-\cos (x)]^{q} \psi_{0}(x), \psi_{0}$ being a positive trigonometric polynomial, when dealing with $\tau$ matrices. In the case of the circulant and Hartley algebras, we must consider the one rank correction displayed in (1.5) in order to force the invertibility. Therefore, by exploiting relation (4.4), we have $f_{0}(x)+c_{0} \chi_{2 \pi \mathbb{Z}}(x)=$ $[1-\cos (x)]^{q} \psi_{0}(x)+c_{0} \chi_{2 \pi \mathbb{Z}}(x)$. In order to get a uniform lower bound to $\alpha_{i} / \beta_{i}$ (in particular to find an upper bound for the left side of (4.8)), it seems convenient to obtain such a factorization for every generating function $f_{i}$. We find the desired result by using Proposition 2.5.

Proposition 4.3. Under the same assumptions of Proposition 2.5, let $q$ be a positive integer and let us suppose $f_{0}(x)=[1-\cos (x)]^{q} \psi_{0}(x)+c_{0} \chi_{2 \pi \mathbb{Z}}(x)$, with $\psi_{0}$ being a positive trigonometric polynomial and with $c_{0}=f_{0}\left(w_{1}^{\left[n_{0}\right]}\right)$ in the circulant and Hartley cases and with $c_{0}=0$ in the $\tau$ case; define also $p_{i}(x)=\sqrt{2}[1+\cos (x)]^{q}+$ 
$d_{i} \chi_{2 \pi \mathbb{Z}}(x)=p(x)+d_{i} \chi_{2 \pi \mathbb{Z}}(x)$ for each $i=0, \ldots, m-1$. Then each generating function $f_{i}$ satisfies $f_{i}(x)=\tilde{f}_{i}(x)+c_{i} \chi_{2 \pi \mathbb{Z}}(x), \tilde{f}_{i}(x)=[1-\cos (x)]^{q} \psi_{i}(x)$ with the sequences $\left\{\psi_{i}\right\}$ and $\left\{c_{i}\right\}$ defined as

$$
\left\{\begin{array}{l}
\psi_{i+1}=\Phi_{q}\left(\psi_{i}\right), \\
c_{i+1}=\frac{1}{2} c_{i} p_{i}^{2}(0),
\end{array} \quad i=0, \ldots, m-1,\right.
$$

where $\Phi_{q}$ is an operator such that

$$
\left[\Phi_{q}(\psi)\right](x)=\frac{1}{2^{q+\frac{1}{2}}}\left[(p \psi)\left(\frac{x}{2}\right)+(p \psi)\left(\pi+\frac{x}{2}\right)\right] .
$$

Moreover, each $\tilde{f}_{i}$ is a trigonometric polynomial that vanishes only at $2 \pi \mathbb{Z}$ with the same order $2 q$ as $f_{0}$.

Proof. Taking into account the expression of $p(x)=\sqrt{2}[1+\cos (x)]^{q}$, the result is a direct consequence of Proposition 2.5 and relation (2.14).

Proposition 4.4. Under the same assumptions of Proposition 2.5, let $q$ be a positive integer and let us suppose $f_{0}(x)=[1-\cos (x)]^{q} \psi_{0}(x)+c_{0} \chi_{2 \pi \mathbb{Z}}(x)$, with $\psi_{0}$ being a positive trigonometric polynomial and with $c_{0}=f\left(w_{1}^{\left[n_{0}\right]}\right)$ in the circulant and Hartley cases and with $c_{0}=0$ in the $\tau$ case; also define $p_{i}(x)=\sqrt{2}[1+\cos (x)]^{q}+$ $d_{i} \chi_{2 \pi \mathbb{Z}}(x)=p(x)+d_{i} \chi_{2 \pi \mathbb{Z}}(x)$ for each $i=0, \ldots, m-1$. Then we can choose numbers $d_{i}$ such that, setting $\tilde{f}_{i}(x)=[1-\cos (x)]^{q} \psi_{i}(x)$, we have $f_{i}(x)=\tilde{f}_{i}(x)+c_{i} \chi_{2 \pi \mathbb{Z}}(x)$ with $c_{i}=0$ in the $\tau$ case and with $c_{i}=f_{i}\left(w_{0}^{\left[n_{i}\right]}\right)=\tilde{f}_{i}\left(w_{1}^{\left[n_{i}\right]}\right)>0$ in the case of circulants and Hartley matrices.

Proof. In the $\tau$ setting we can choose $d_{i}=0$. Therefore, since $c_{0}=0$ there is nothing to prove. In the remaining cases, the result follows from the relations $f_{i}(0)=\tilde{f}_{i}(0)+c_{i}=c_{i}, c_{i+1}=\frac{1}{2} c_{i} p_{i}^{2}(0)=\frac{1}{2} c_{i}\left(\sqrt{2} 2^{q}+d_{i}\right)^{2}$ and from the fact that $c_{0}=f\left(w_{1}^{\left[n_{0}\right]}\right)$ : more specifically we have

$$
d_{i}=\sqrt{\frac{2 f_{i+1}\left(\frac{2 \pi}{n_{i+1}}\right)}{f_{i}\left(\frac{2 \pi}{n_{i}}\right)}}-\sqrt{2} 2^{q} .
$$

Propositions 4.3 and 4.4 will allow us to find bounds for the constants $\alpha_{i}$ and $\beta_{i}$ involved in (2.11).

We now have the tools for defining a really recursive V-cycle technique (as explained in Proposition 4.5) and for proving that we can satisfy the approximation property (Proposition 4.6).

Proposition 4.5. Let $A_{i}=\mathcal{A}_{n_{i}}\left(f_{i}\right), P_{i+1}^{i}=K_{i+1}^{i} \mathcal{A}_{n_{i}}\left(p_{i}\right)$, with $f_{i}$ being a nonnegative polynomial (also even in the $\tau$ case) and $p_{i}$ satisfying conditions (4.2) (also even in the $\tau$ case).

1. The projected matrix $A_{i+1}$ coincides with $\mathcal{A}_{n_{i+1}}\left(f_{i+1}\right)$, where $f_{i+1}$ has the expression reported in (2.13).

2. If $x_{0} \in[-\pi, \pi]$ is a zero of $f_{i}(x)$ then $f_{i+1}$ has a corresponding zero $y_{0}=2 x_{0}$.

3. $f_{i}$ and $f_{i+1}$ have the same number of zeros, i.e., for any zero $y_{0} \in[-\pi, \pi]$ of $f_{i+1}$ there exists a unique zero of $f_{i}$ such that the relations in the preceding item holds true.

4. The order of the zero $y_{0}$ of $f_{i+1}$ is exactly the same as the one of the zero $x_{0}$ of $f_{i}$ so that at the lower level the new projector is easily defined in the same way. 
Proposition 4.6. For every $i=0, \ldots, m-1$, let $A_{i}=\mathcal{A}_{n_{i}}\left(f_{i}\right)$ with $\mathcal{A} \in$ $\{\mathcal{C}, \mathcal{H}, \tau\}$ as in (1.4) and let $f_{i}$ be as in Proposition 4.3. Let $P_{i+1}^{i}=K_{i+1}^{i} \cdot \mathcal{A}_{n_{i}}\left(p_{i}\right)$ and let us define $C G C_{i}$ as in (2.5). Assume that $p_{i}(x)=\tilde{p}_{i}(x)+d_{i} \chi_{2 \pi \mathbb{Z}}(x)$ with $\tilde{p}_{i}$ fulfilling (4.2) (also even in the $\tau$ case) and with $d_{i}$ as in Proposition 4.4 (for instance, take $\tilde{p}_{i}(x)=\sqrt{2}[1+\cos (x)]^{q}$ as in Proposition 4.3). Then for every $i=0, \ldots, m-1$, there exists a real and positive value $\beta_{i}$ such that

$$
\left\|C G C_{i} \mathbf{x}\right\|_{A_{i}}^{2} \leqslant \beta_{i}\|\mathbf{x}\|_{A_{i}^{2}}^{2}, \quad \mathbf{x} \in \mathbb{C}^{n_{i}} .
$$

Proof. Relation (4.6) can be rewritten in matrix form as

$$
C G C_{i}^{H} A_{i} C G C_{i} \leqslant \beta_{i} A_{i}^{2}
$$

By straightforward calculation we have $C G C_{i}^{H} A_{i} C G C_{i}=A_{i} C G C_{i}$, and hence (4.6) holds if and only if $A_{i} C G C_{i} \leqslant \beta_{i} A_{i}^{2}$ is satisfied. By multiplying from both the sides by $A_{i}^{-1 / 2}$ we get

$$
I_{n_{i}}-A_{i}^{1 / 2}\left(P_{i+1}^{i}\right)^{H}\left[P_{i+1}^{i} A_{i}\left(P_{i+1}^{i}\right)^{H}\right]^{-1} P_{i+1}^{i} A_{i}^{1 / 2} \leqslant \beta_{i} A_{i}
$$

and then, by defining $\hat{P}_{i+1}^{i}=P_{i+1}^{i} \cdot A_{i}^{1 / 2}$, we infer

$$
I_{n_{i}}-\left(\hat{P}_{i+1}^{i}\right)^{H}\left[\hat{P}_{i+1}^{i}\left(\hat{P}_{i+1}^{i}\right)^{H}\right]^{-1} \hat{P}_{i+1}^{i} \leqslant \beta_{i} A_{i},
$$

where $\hat{P}_{i+1}^{i}=K_{i+1}^{i} \cdot \mathcal{A}_{n_{i}}\left(\hat{p}_{i}(x)\right)$ with $\hat{p}_{i}(x)=p_{i}(x) \cdot f_{i}^{1 / 2}(x)$. We notice that (4.7) can be found in [31] while showing the TGM approximation property for the circulant algebra, and is also contained in the proof of Lemma 3.2 in [27], while showing the same property in the $\tau$ algebra (the Hartley case is totally analogous to circulants). Thus, by performing a block diagonalization of all the involved matrices (see Lemma 3.2 in [27]), to have (4.6), it is enough to prove

$$
\frac{1}{\hat{p}_{i}^{2}(x)+\hat{p}_{i}^{2}(x+\pi)}\left[\begin{array}{cc}
\hat{p}_{i}^{2}(\pi+x) & -\hat{p}_{i}(x) \hat{p}_{i}(\pi+x) \\
-\hat{p}_{i}(x) \hat{p}_{i}(\pi+x) & \hat{p}_{i}^{2}(x)
\end{array}\right] \leqslant \beta_{i}\left[\begin{array}{cc}
f_{i}(x) & \\
& f_{i}(\pi+x)
\end{array}\right]
$$

for every $x \in \bigcup_{j \in \mathcal{I}_{n_{i+1}}}\left\{\frac{1}{2} w_{j}^{\left[n_{i+1}\right]}\right\}$, and once again, by following the proof of Lemma 3.2 in [27], we deduce that (4.6) is guaranteed if

$$
\frac{1}{\hat{p}_{i}^{2}(x)+\hat{p}_{i}^{2}(\pi+x)} \cdot\left(\frac{\hat{p}_{i}^{2}(x)}{f_{i}(\pi+x)}+\frac{\hat{p}_{i}^{2}(\pi+x)}{f_{i}(x)}\right) \leqslant \beta_{i} \quad \forall x \in \bigcup_{j \in \mathcal{I}_{n_{i+1}}}\left\{\frac{w_{j}^{\left[n_{i+1}\right]}}{2}\right\} .
$$

Therefore, in terms of the involved generating functions, we obtain that the following conditions have to be satisfied:

$$
\frac{1}{\frac{p_{i}^{2}(x)}{f_{i}(\pi+x)}+\frac{p_{i}^{2}(\pi+x)}{f_{i}(x)}} \cdot\left(\frac{p_{i}^{2}(x)}{f_{i}^{2}(\pi+x)}+\frac{p_{i}^{2}(\pi+x)}{f_{i}^{2}(x)}\right) \leqslant \beta_{i} \quad \forall x \in \bigcup_{j \in \mathcal{I}_{n_{i+1}}}\left\{\frac{w_{j}^{\left[n_{i+1}\right]}}{2}\right\} .
$$


Finally, we observe that the first inequality in (4.2) implies the uniform boundedness (with respect to $n_{i}$ and to $x$ ) of the term

$$
\frac{1}{\frac{p_{i}^{2}(x)}{f_{i}(\pi+x)}+\frac{p_{i}^{2}(\pi+x)}{f_{i}(x)}}
$$

while the second inequality in (4.2) implies the uniform boundedness (with respect to $n_{i}$ and to $x$ ) of the term

$$
\left(\frac{p_{i}^{2}(x)}{f_{i}^{2}(\pi+x)}+\frac{p_{i}^{2}(\pi+x)}{f_{i}^{2}(x)}\right)
$$

and therefore the proof is over with $\beta_{i}$ being the products of the two constants realizing the above mentioned bounds.

Remark 4.7. The statement in Proposition 4.6, namely relation (4.6), holds unchanged in the more general setting where the zero $x_{0}$ is not 0 . It is sufficient to show that $A_{i}$ is nonsingular and indeed the rest of the proof of Proposition 4.6 will remain the same. Let $A_{i}=\mathcal{A}_{n_{i}}\left(f_{i}\right)$ with $\mathcal{A} \in\{\mathcal{C}, \mathcal{H}, \tau\}$ for $i=0, \ldots, m$ and let $x_{0}$ be the unique zero of $f_{0}$ in $[0,2 \pi)$ (in the $\tau$ case $f_{0}$ is even and has also a zero at $\left.2 \pi-x_{0}\right)$.

1. If $x_{0} \notin \bigcup_{j \in \mathcal{I}_{n_{0}}}\left\{w_{j}^{\left[n_{0}\right]}\right\}$ (also $2 \pi-x_{0} \notin \bigcup_{j \in \mathcal{I}_{0}}\left\{w_{j}^{\left[n_{0}\right]}\right\}$ in the $\tau$ case), then, by Proposition 4.5, $f_{i}$ vanishes only at $x_{i} \notin \bigcup_{j \in \mathcal{I}_{n_{i}}}\left\{w_{j}^{\left[n_{i}\right]}\right\}$ (also at $2 \pi-$ $x_{i} \notin \bigcup_{j \in \mathcal{I}_{n_{i}}}\left\{w_{j}^{\left[n_{i}\right]}\right\}$ in the $\tau$ case) and therefore $A_{i}$ is nonsingular for every $i=0, \ldots, m$.

2. If $\exists j \in \mathcal{I}_{n_{0}}: x_{0}=w_{j}^{\left[n_{0}\right]}$ (also $2 \pi-x_{0}=w_{n+1-j}^{\left[n_{0}\right]}$ in the $\tau$ case), we proceed as in the case $x_{0}=0$ (see Propositions 4.3 and 4.4). We fix $\tilde{f}_{i}(x)=(1-\cos (x-$ $\left.\left.x_{i}\right)\right)^{q}, c_{0}=\min \left\{f_{0}\left(w_{j-1}^{\left[n_{0}\right]}\right), f_{0}\left(w_{j+1}^{\left[n_{0}\right]}\right)\right\}$ and $f_{i}(x)=\tilde{f}_{i}(x)+c_{i} \chi_{x_{0}+2 \pi \mathbb{Z}}(x)$ (also $f_{i}(x)=\tilde{f}_{i}(x)+c_{i} \chi_{-x_{0}+2 \pi \mathbb{Z}}(x)$ in the $\tau$ case), then, by Proposition 4.5, $\tilde{f}_{i}$ vanishes at $x_{i}=w_{j}^{\left[n_{i}\right]}$ (also at $w_{n+1-j}^{\left[n_{0}\right]}$ in the $\tau$ case) for $i=0, \ldots, m$. The quantities $c_{i}, d_{i}$ and $p_{i}(x)$ are calculated as in Propositions 4.3 and 4.4, where 0 is replaced by $x_{0}$. In this case $A_{i}$ is again nonsingular for every $i=0, \ldots, m$.

4.3. MGM optimal convergence (i.e., verification of the inf - min condition). In Propositions 4.1 and 4.6 we have proven that for every $i$ (independent of $n=n_{0}$ ) the constants $\alpha_{i}$ and $\beta_{i}$ are absolute values not depending on $n=n_{0}$ but only depending on the functions $f_{i}$ and $p_{i}$. However, in order to fulfill conditions (2.11a) and (2.11b) with $\delta$ independent of $n$ (which in turn imply the MGM optimal convergence by Theorem 2.3), we should prove the following inf-min condition:

$$
\delta=\inf _{n} \min _{1 \leqslant m \leqslant \phi(n)} \min _{0 \leqslant i \leqslant m} \frac{\alpha_{i}}{\beta_{i}}=\inf _{n} \min _{0 \leqslant i \leqslant \phi(n)} \frac{\alpha_{i}}{\beta_{i}}>0 .
$$

Here $\phi(n)$ is the maximal number of possible recursion levels and it equals $\log _{2}(n)$ for circulants and Hartley matrices and coincides with $\log _{2}(n+1)$ for $\tau$ matrices. In the following we will consider the case where the trigonometric polynomial $f_{0}$ is positive in the interval $(0,2 \pi)$ and takes the zero value at the origin, and we will demonstrate the inf-min condition (4.9).

In the following, for a given function $f$, we will write $M_{f}=\sup _{x}|f|, m_{f}=\inf _{x}|f|$ and $\mu_{\infty}(f)=M_{f} / m_{f}$. In (2.11a) we simply find $\alpha_{i}\left(\omega_{i}^{*}=\left\|f_{i}\right\|_{\infty}^{-1}\right)=\left\|f_{i}\right\|_{\infty}^{-1} \geqslant$ 
$1 /\left(2^{q} M_{\psi_{i}}\right)$, while (from $p(x)=\sqrt{2}[1+\cos (x)]^{q}$ it follows that the range of $p(x)+$ $p(\pi+x)$ is $\left.\left[\sqrt{2} \cdot 2, \sqrt{2} \cdot 2^{q}\right]\right)$ in order to get an upper bound for the left-hand side in (4.8), if $x \in(0,2 \pi)$ we obtain

$$
\frac{\frac{p_{i}^{2}(x)}{f_{i}^{2}(\pi+x)}+\frac{p_{i}^{2}(\pi+x)}{f_{i}^{2}(x)}}{\frac{p_{i}^{2}(x)}{f_{i}(\pi+x)}+\frac{p_{i}^{2}(\pi+x)}{f_{i}(x)}}=\sqrt{2} \frac{\frac{1}{\psi_{i}^{2}(\pi+x)}+\frac{1}{\psi_{i}^{2}(x)}}{\frac{p_{i}(x)}{\psi_{i}(\pi+x)}+\frac{p_{i}(\pi+x)}{\psi_{i}(x)}} \leqslant \sqrt{2} \frac{\frac{2}{m_{\psi_{i}}^{2}}}{\frac{p(x)+p(\pi+x)}{M_{\psi_{i}}}} \leqslant \frac{M_{\psi_{i}}}{m_{\psi_{i}}^{2}}
$$

so $\beta_{i}=M_{\psi_{i}} / m_{\psi_{i}}^{2}$ works fine, while if $x=0$ we have also to require $1 / f_{i}(\pi) \leqslant \beta_{i}$ to ensure that inequality (2.11b) is satisfied: more precisely, at $x=0$ (by (4.8), the case is of interest only for circulants and Hartley matrices since $x=0$ is not a grid point for $\tau$ matrices), we have

$$
\begin{aligned}
p_{i}(0) & =\sqrt{2} 2^{q}+d_{i}, \\
f_{i}(0) & =c_{i}>0, \\
p_{i}(\pi) & =0 \\
f_{i}(\pi) & >0
\end{aligned}
$$

and therefore (4.8) holds at $x=0$ with any constant $\beta_{i}$ such that $1 / f_{i}(\pi) \leqslant \beta_{i}$. Since $\left(M_{\psi_{i}} / m_{\psi_{i}}^{2}\right) \cdot f_{i}(\pi) \geqslant f_{i}(\pi) / m_{\psi_{i}} \geqslant 1$, it follows that $\beta_{i}^{*}=M_{\psi_{i}} / m_{\psi_{i}}^{2}$ is the best value. As a consequence, it follows that

$$
\frac{\alpha_{i}}{\beta_{i}} \geqslant \frac{1}{2^{q} M_{\psi_{i}}} \cdot \frac{m_{\psi_{i}}^{2}}{M_{\psi_{i}}}=\frac{1}{2^{q} \mu_{\infty}^{2}\left(\psi_{i}\right)}
$$

Therefore, to enforce the inf-min condition (4.9), it is enough to prove the existence of an absolute constant $L$ such that $\mu_{\infty}\left(\psi_{i}\right) \leqslant L<+\infty$ uniformly to deduce that $\left\|M G M_{0}\right\|_{A_{0}} \leqslant \sqrt{1-2^{-q} L^{-2}}<1$ : the latter follows from the next proposition.

Proposition 4.8. Under the same assumptions of Proposition 4.3, let $\psi_{0}$ be a positive polynomial and let us define $\psi_{i}=\left[\Phi_{q}\right]^{i}(\psi)$ for every $i \in \mathbb{N}$, where $\Phi_{q}$ is the linear operator defined as in (4.5). Then there exists a positive polynomial $\psi_{\infty} \in \mathbb{R}_{q-1}$ such that $\psi_{i}$ uniformly converges to $\psi_{\infty}$, and moreover there exists a positive real number $L$ such that $\mu_{\infty}\left(\psi_{i}\right) \leqslant L$ for every $i \in \mathbb{N}$.

Proof. The proof is organized into two parts.

Part A. From the definition of the operator $\Phi_{q}$ in (4.5) and from the assumptions on the polynomials $p_{i}$ (see Proposition 4.3), it follows that the positivity (and the boundedness) of $\psi_{0}$ implies the positivity (and the boundedness) of $\psi_{i}$ for every $i \in \mathbb{N}$, i.e., there exist positive constants $L_{i}$ such that

$$
\mu_{\infty}\left(\psi_{i}\right) \leqslant L_{i}
$$

Part B. We give a linear algebra proof of the fact that, starting a polynomial $\psi_{0}$ such that $\psi_{0}(0)>0$, the operator $\Phi_{q}$ in (4.5) has a strictly positive fixed point belonging to $\mathbb{R}_{q}$, and therefore there exists a constant $L_{\infty}$ such that

$$
\lim _{i \rightarrow \infty} \mu_{\infty}\left(\psi_{i}\right)=L_{\infty}
$$

Therefore the second result $\left(\mu_{\infty}\left(\psi_{i}\right) \leqslant L\right.$ for $i \in \mathbb{N}$ and for a pure constant $\left.L>0\right)$ will be a straightforward consequence of (4.11) and of (4.12) which, in turn, is a 
consequence of the uniform convergence of the sequence $\psi_{i}$ and of the fact that its limit is a strictly positive function. The latter is what we are going to prove.

From (2.14) it follows that $f_{i} \in \mathbb{R}_{T_{i}}$ with $T_{i+1}=q+\left\lfloor\frac{T_{i}}{2}\right\rfloor$ if $f_{0} \in \mathbb{R}_{T_{0}}$, and hence, since $f_{i}=\psi_{i}[1-\cos (x)]^{q}$, there exists an index $j \in \mathbb{N}$ such that $\psi_{i} \in \mathbb{R}_{q}$ when $i \geqslant j$ and we can suppose $\psi_{i} \in \mathbb{R}_{q}$. We demonstrate a bit stronger result, i.e.,

$$
\left\{\begin{array}{l}
\psi \in \mathbb{R}_{q} \\
\psi(0)>0
\end{array} \quad \Rightarrow \quad \exists \psi^{*} \in \mathbb{R}_{q}:\left[\Phi_{q}\right]^{i}(\psi) \stackrel{\text { uniformly }}{\longrightarrow} \psi^{*} .\right.
$$

As $\Phi_{q}$ is linear, by expressing the problem in the basis $\left\{e^{-\mathbf{i} q x} ; \ldots ; e^{\mathbf{i} q x}\right\}$ and by denoting by $\bar{\Phi}_{q}$ the matrix representing $\Phi_{q}$ in such a basis, the preceding implication is equivalent to proving that

$$
\left\{\begin{array}{l}
\mathbf{a} \in \mathbb{C}^{2 q+1} \\
\sum_{j=1}^{2 q+1} a_{j}>0
\end{array} \quad \Rightarrow \quad \exists \mathbf{a}^{*} \in \mathbb{C}^{2 q+1}:\left[\bar{\Phi}_{q}\right]^{i} \mathbf{a} \longrightarrow \mathbf{a}^{*} .\right.
$$

If (4.13) holds true, then there exists $\psi^{*} \in \mathbb{R}_{q}$ (defined by $\psi^{*}(x)=\sum_{|j| \leqslant q} a_{j+q+1}^{*} e^{\mathbf{i} j x}$ ) such that $\psi_{i} \longrightarrow \psi^{*}$ uniformly and $\Phi_{q}\left(\psi^{*}\right)=\psi^{*}$. Moreover, from the assumptions on $p_{i}$ (see Proposition 4.3), we have $p_{i}(\pi)=0, p(0)=2^{q+\frac{1}{2}}$ and therefore, by (4.5), we have

$$
\psi_{i+1}(0)=\Phi_{q}\left(\psi_{i}\right)(0)=\frac{p(0)}{2^{q+\frac{1}{2}}} \psi_{i}(0)=\psi_{i}(0) .
$$

Thus $\psi^{*}(0)=\psi_{0}(0)>0$. The last condition ensures $\psi^{*}>0$, because, from $\psi^{*}(\bar{x})=0$ and from the definition of $\Phi_{q}(\cdot)$, it follows $\psi^{*}\left(\bar{x} / 2^{s}\right)=0$ for every $s \in \mathbb{N}$ (use (4.5)), and this is clearly impossible because $\psi^{*}$ is continuous and therefore

$$
\lim _{s \rightarrow \infty} \psi^{*}\left(\bar{x} / 2^{s}\right)=\psi^{*}(0)>0 .
$$

We still have to show (4.13). In actuality, (4.13) follows if we demonstrate that $\bar{\Phi}_{q}$ has one eigenvalue equal to 1 with algebraic multiplicity 1 and positive eigenvector $\mathbf{a}^{*}$, while all the other eigenvalues $\lambda_{i}$ enjoy the relation $\left|\lambda_{i}\right|<1$ : to this aim we will use the Perron-Frobenius theorem $[19,36]$. Let us look at $\bar{\Phi}_{q}$. We define $b_{j}^{(q)},|j| \leqslant q$, as the Fourier coefficient of $\frac{1}{2^{q+\frac{1}{2}}} p(x)$ (i.e., of $\cos ^{2 q}(x / 2)$ ):

$$
b_{j}^{(q)}=\frac{1}{2 \pi} \int_{-\pi}^{\pi} \frac{1}{2^{q+\frac{1}{2}}} p(x) e^{\mathbf{i} j x} d x=\frac{(2 q) !}{4^{q}(q-j) !(q+j) !}>0, \quad b_{j}^{(q)}=b_{-j}^{(q)} .
$$

It holds that $p(x) e^{\mathbf{i} k x}=\sum_{j=k-q}^{k+q} b_{j-k}^{(q)} e^{\mathbf{i} j x}$ and hence $\bar{\Phi}_{q}$ has (by (2.14)) the following matrix form:

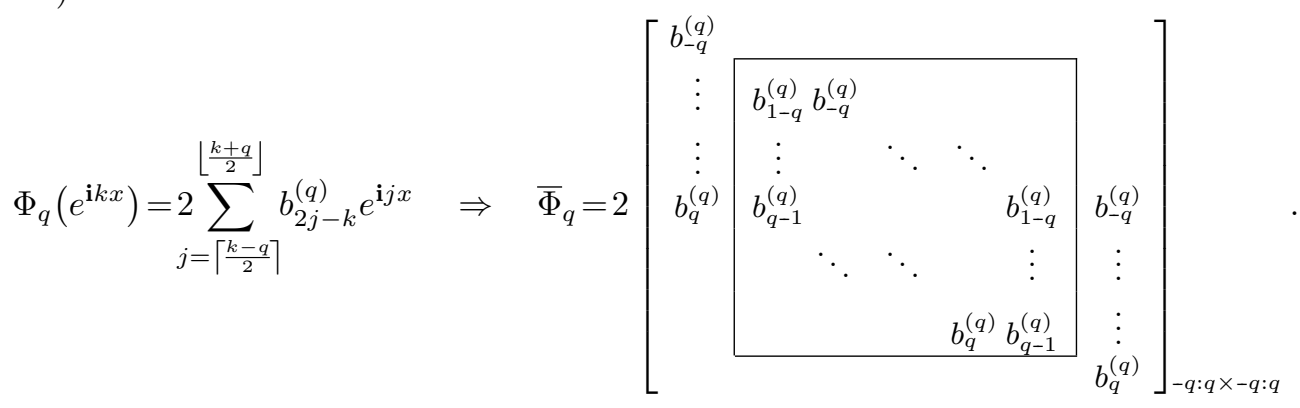


Nonvanishing entries in the $k$ th column are the coefficients $2 b_{j}^{(q)}$ such that $j \equiv$ $k(\bmod 2)$. We observe $b_{q}^{(q)}=b_{-q}^{(q)}=4^{-q}<1$ and then we have only to check the behavior of the eigenvalues of the submatrix $M$ with both indices ranging from $-q+1$ to $q-1$ (this matrix is the one displayed in the inner box of (4.14)). The corresponding analysis is now straightforward: the vector of all ones is an eigenvector of $M^{T}$ related to the eigenvalue 1 (because of the left side in (4.14)); $\|M\|_{\infty}=1$, $M_{i j} \geqslant 0$ and $M$ is irreducible. Finally, the result follows from the Perron-Frobenius theorem applied to the matrix $M$.

We remark that the previous result on the limit polynomial $\psi^{*}$ can be refined a little bit. Indeed, it belongs to $\mathbb{R}_{q-1}$ (instead of $\mathbb{R}_{q}$ ) since the eigenvector $\mathbf{a}^{*}$ of $\bar{\Phi}_{q}$ related to the dominating eigenvalue $\lambda=1$ is of the form

$$
\left(\begin{array}{c}
0 \\
\hat{\mathbf{a}} \\
0
\end{array}\right) \text {, }
$$

where $\hat{\mathbf{a}}$ is the positive eigenvector of $M$ associated with the dominating eigenvalue $\lambda=1$.

THEOREM 4.9. Let $f$ be a trigonometric polynomial, positive in $(0,2 \pi)$ and vanishing at 0 with order $2 q$ (also even in the $\tau$ case); let us fix integers $k_{0}, m$ such that $0<m<k_{0}$, and let us define $n_{i}, i=0, \ldots, m$, as in (2.12).

For every $i=0, \ldots, m-1$, define also the following quantities: $p_{i}(x)=\sqrt{2}[1+$ $\cos (x)]^{q}+d_{i} \chi_{2 \pi \mathbb{Z}}(x)$ with $d_{i}$ as Proposition $4.4, K_{i+1}^{i}$ as in (2.12), $P_{i+1}^{i}=K_{i+1}^{i} \mathcal{A}_{n_{i}}\left(p_{i}\right)$ with $\mathcal{A} \in\{\mathcal{C}, \mathcal{H}, \tau\}$ as in (1.4), and $\mathcal{R}_{i}$ as in (2.2) with $R_{i}=I_{n_{i}}-A_{n_{i}} /\left\|f_{i}\right\|_{\infty}$.

If we set $A_{0}=\mathcal{A}_{n_{0}}\left(f+c_{0} \chi_{2 \pi \mathbb{Z}}\right)$ with $c_{0}=f\left(w_{1}^{\left[n_{0}\right]}\right)$ in the circulant and Hartley cases and with $c_{0}=0$ in the $\tau$ case, and we consider $\mathbf{b} \in \mathbb{C}^{n_{0}}$, then the $V$-cycle algorithm defined in (2.1) converges to the solution of $A_{0} \mathbf{x}=\mathbf{b}$ and is optimal (in the sense of Definition 1.1).

Proof. From Proposition 4.2 we know that

$$
f(x)=[1-\cos (x)]^{q} \cdot \psi(x)
$$

for some positive polynomial $\psi$. Now, it is enough to observe that the MGM optimal convergence stated in Theorem 2.3 is implied by the inf-min condition (4.9) which, in turn, by (4.10), is implied by the uniform boundedness of the quantities $\mu_{\infty}\left(\psi_{i}\right)$ and the latter has been proven in Proposition 4.8.

4.4. The case of a unique zero at $x_{0} \neq 0$ : Circulant and Hartley algebras. We now consider matrices belonging to the circulant and Hartley algebras, whose generating function $f_{0}$ vanishes in a generic point $x_{0}$. We remark that Proposition 4.2 ensures $f_{0}=\left[1-\cos \left(x-x_{0}\right)\right]^{q} \psi_{0}\left(x-x_{0}\right)$. Consequently, as in the previous situation $\left(x_{0}=0\right)$, we obtain a similar result.

Proposition 4.10. Under the same assumptions of Proposition 2.5, let $f_{0}(x)=\left[1-\cos \left(x-x_{0}\right)\right]^{q} \psi_{0}\left(x-x_{0}\right)$ with $q$ positive and integer and let $\psi_{0}$ be a positive trigonometric polynomial. By defining $x_{i+1}=2 x_{i}(\bmod 2 \pi)$ and $p_{i}(x)=$ $\sqrt{2}\left[1+\cos \left(x-x_{i}\right)\right]^{q}$ for every $i=0, \ldots, m-1$, we deduce that the generating functions $f_{i}$ enjoy the following relation:

$$
f_{i}(x)=\left[1-\cos \left(x-x_{i}\right)\right]^{q} \psi_{i}\left(x-x_{i}\right) .
$$

Proof. It suffices to write $f_{i+1}(x)=\frac{1}{2}\left[\left(p_{i}^{2} f_{i}\right)\left(\frac{x-2 x_{i}}{2}\right)+\left(p_{i}^{2} f_{i}\right)\left(\pi+\frac{x-2 x_{i}}{2}\right)\right]$ and to apply the statement contained in Proposition 4.3. 
In such a situation the functions $f_{i}$ will not converge, but the values $\mu_{\infty}\left(\psi_{i}\right)$ remain unchanged and the latter is enough to prove the MGM optimal convergence (see (4.10)).

4.5. The case of a unique zero at $x_{0} \neq 0$ : the $\tau$ algebra. Since the generating function $f_{0}$ must be also even, it follows that the unicity of the zero $x_{0} \neq 0$ implies that $f_{0}$ has to vanish at $x_{0}=\pi$. In addition, it is worth mentioning that when the coefficient matrix is $A=\tau_{n_{0}}\left(f_{0}\right)$ and $f_{0}(\pi)=0$ and is positive elsewhere in $(0,2 \pi)$, from relation $(2.13)$, it follows that the function $f_{1}$ has a unique zero at 0 . Since the MGM optimality, for functions having a unique zero at the origin, has been proven (see Theorem 4.9), it easily follows that the MGM optimal convergence stands for the case of a unique zero at $\pi$. Indeed, looking at the MGM applied to $\tau_{n_{1}}\left(f_{1}\right)$, it holds that (2.9) is satisfied with

$$
\bar{\delta} \text { independent of } n_{1}=\frac{n_{0}-1}{2} \quad \forall i=1, \ldots m-1 .
$$

Therefore, Theorem 2.3 holds with $\delta=\min \left\{\delta_{0}, \bar{\delta}\right\}$, which is constant and independent of $n_{0}$, i.e., the MGM is optimal.

We point out that the case of generating function that vanishes at $\pi$ with respect to each variable is particularly important in applications. In fact, certain integral equations when discretized lead to matrices belonging to this class. For instance, the signal restoration leads to the case of $f(\pi)=0$, while for the super-resolution problem and image restoration we have $f(\pi, \pi)=0$ [8]. Therefore, it is interesting to stress that the application of the V-cycle algorithm is such that a discretized integral problem is projected, at the lower level, into another which is spectrally and structurally equivalent to a discretized differential problem.

Finally, we observe that the case of two zeros $x_{0}$ and $2 \pi-x_{0}$ for $x_{0} \notin\{0, \pi\}$ is not different from the case of a unique zero since Proposition 4.2 holds with $\left[\cos \left(x_{0}\right)-\cos (x)\right]^{2 q}$ in place of $\left[1-\cos \left(x-x_{0}\right)\right]^{q}$ and (4.10) is satisfied as well.

5. The multilevel case. We briefly describe our choice of projectors and smoothers in the multilevel case and we indicate how to generalize the proof of MGM optimal convergence (for the TGM the optimality has been already proven in $[27,31]$ ).

The smoothing iteration is formally defined as in the unilevel case. The projectors are constructed as $U_{i+1}^{i} \mathcal{A}_{n_{i}}\left(p_{i}\right)$, where $n_{i}=\left(\left(n_{i}\right)_{1}, \ldots,\left(n_{i}\right)_{d}\right)$, the polynomial $p_{i}$ is $d$ variate polynomial and the matrix $U_{i+1}^{i}$ is defined as $K_{i+1}^{i, 1} \otimes \cdots \otimes K_{i+1}^{i, d}$ with $K_{i+1}^{i, j}$ being the $\left(n_{i+i}\right)_{j} \times\left(n_{i}\right)_{j}$ unilevel cutting matrix related to $\mathcal{A}$ explicitly given in (2.12). If the coefficient matrix is $\mathcal{A}_{n_{i}}\left(f_{i}\right)$ with $f_{i}$ having a unique zero at $x_{0}$ of order $2 q$, the matrix $\mathcal{A}_{n_{i}}\left(p_{i}\right)$ is chosen with $p_{i}$ such that

$$
\limsup _{x \rightarrow x_{0}}\left|\frac{p_{i}(\hat{x})}{f_{i}(x)}\right|<+\infty, \quad \hat{x} \in M(x), i=0, \ldots, m-1,
$$

where

$$
0<\sum_{\hat{x} \in M(x) \cup\{x\}} p_{i}^{2}(\hat{x}), \quad i=0, \ldots, m-1,
$$

with $M(x)$ being the set of the "mirror points" of $x$ introduced for $d=2$ in [16]. A formal definition is the following: $\hat{x} \in M(x)$ if and only if $\hat{x} \neq x$ and $\forall j=1, \ldots, d$ it holds $\hat{x}_{j} \in\left\{(x)_{j}, \pi+(x)_{j}\right\}$. For $d=1$, it is evident that the unique mirror point 
is $\pi+x$, while in the general case the cardinality of $M(x)$ is $2^{d}-1$. Notice that $\forall \hat{x} \in M(x)$ we have $M(\hat{x})=\{M(x) \backslash\{\hat{x}\}\} \cup\{x\}$.

If $f_{i}$ has more than one zero in $[0,2 \pi]^{d}$ then the corresponding polynomial $p_{i}$ will be the product of the basic polynomials satisfying (5.1) and (5.2) for any single zero.

Remark 5.1. In the case of more than one zero, relation (5.2) imposes some restrictions on the zeros of $f_{0}$. First, the zeros of $f_{0}$ should be of finite order (by (5.1)) and this is true in the case of a unique zero, too. Second, if $x^{0}$ is a zero of $f_{0}$ then $f(\hat{x})>0$ for any $\hat{x} \in M\left(x^{0}\right)$; otherwise relationship (5.2) cannot be satisfied with any polynomial $p_{0}$. As in the unidimensional case the second restriction can be removed by changing the "form" of the projection that is its smaller dimension.

Proposition 5.2. Let $A_{i}=\mathcal{A}_{n_{i}}\left(f_{i}\right), P_{i+1}^{i}=U_{i+1}^{i} \mathcal{A}_{n_{i}}\left(p_{i}\right)$, with $f_{i}$ being a nonnegative polynomial (also even in the $\tau$ case) and $p_{i}$ satisfying conditions (5.1) and (5.2) (also even in the $\tau$ case).

1. The projected matrix $A_{i+1}$ coincides with $\mathcal{A}_{n_{i+1}}\left(f_{i+1}\right)$, where

$$
2^{d} \hat{f}_{i+1}(x)=\sum_{\hat{x} \in M(x / 2) \cup\{x / 2\}} f_{i}(\hat{x}) p_{i}^{2}(\hat{x})
$$

for $x=\left(x_{1}, \ldots, x_{d}\right) \in[-\pi, \pi]^{d}$.

2. If $x^{0} \in[-\pi, \pi]^{d}$ is a zero of $f_{i}(x)$, then $f_{i+1}$ has a corresponding zero $y^{0} \in$ $[-\pi, \pi]^{d}$ where $y_{j}^{0}=2 x_{j}^{0}$ with $j=1, \ldots, d$.

3. $f_{i}$ and $f_{i+1}$ have the same number of zeros, i.e., for any $y^{0} \in[-\pi, \pi]^{d}$ zero of $f_{i+1}$ there exists a unique zero of $f_{i}$ such that the relations in the preceding item holds true.

4. The order of the zero $y^{0}$ of $f_{i+1}$ is exactly the same as the one of the zero $x^{0}$ of $f_{i}$ so that at the lower level the new projector is easily defined in the same way.

The preceding proposition gives us the necessary tools for talking about the MGM optimal convergence. Indeed it is easy to verify that the proofs of Propositions 4.1 and 4.6 are directly generalized to the multilevel setting. The difficult part concerns relation (4.10), which is strongly based on the factorization result of Proposition 4.2. In actuality, we notice that relation (4.4) is inherently one-dimensional so that the complete multilevel proof could require a different tool at this point of the reasoning: in this respect, very recently, a substantial step has been made by the first two authors by considering an additive representation of the symbols (for more details see [2]).

6. MGM techniques for multilevel Toeplitz matrices. We first observe that the discretization of elliptic boundary value problems with constant coefficients and many image restoration problems lead to Toeplitz structures in which the symbol $f=f_{0}$ is polynomial, nonnegative with isolated zeros, and even (with respect to every direction if $f_{0}$ is multivariate). The latter property suggests that the right starting point for generalizing the V-cycle algorithm to Toeplitz structures should be the MGM for $\tau$ matrices (see also the beginning of Hackbush's book [17]).

In the following, we generalize the $\mathrm{V}$-cycle techniques previously defined for the (multilevel) $\tau$ algebra to the (multilevel) Toeplitz class using the relation (1.2) which characterizes any Toeplitz matrix as its natural $\tau$ preconditioner plus a Hankel correction. In [27] the author presents three different choices of $P_{i+1}^{i}$ when the coefficient matrix $A_{n_{0}}\left(f_{0}\right)$ is Toeplitz:

(A) $P_{i+1}^{i}=K_{i+1}^{i} T_{n_{i}}\left(p_{i}\right)$ 
(B) $P_{i+1}^{i}=K_{i+1}^{i} \tau_{n_{i}}\left(p_{i}\right)$

(C) $P_{i+1}^{i}=K_{i+1}^{i}\left[t_{i}\right] T_{n_{i}}\left(p_{i}\right), \quad i=0, \ldots, m-1$.

Here $p_{i}$ is the projection trigonometric polynomial defined via the same conditions as in the $\tau$ algebra case for every level $i=0, \ldots, m-1$. For the TGM we have only $i=0$ and $p_{0}$ is such that the conditions (2.15) are satisfied. On the other hand, for the multigrid algorithm (see section 4 ), the polynomials $p_{i}$ are chosen in such a way that the stronger conditions (4.2) are satisfied. The choice (A) is the most natural, but unfortunately the lower level matrix $A_{n_{i+1}}=P_{i+1}^{i} A_{n_{i}}\left(P_{i+1}^{i}\right)^{H}$ is not Toeplitz unless the degree of $p_{i}$ does not exceed 1 . With the choice (B), the optimality of the TGM with $A_{n_{0}}=T_{n_{0}}\left(f_{0}\right)$ has been proven in [27]. With the choice (C), for every $t \geqslant 0$, the cutting matrix $K_{i+1}^{i}[t]$ coincides with the submatrix of $K_{i+1}^{i}$ obtained by deleting its first and last $t$ rows with $t=b-1$, where $b$ is the degree of $p_{i}$ that is equal to the degree of $p_{0}$ for $i=0, \ldots, m-1$ (according to Propositions 4.5 and 5.2, at each level the order of the zeros of $f_{i}$ is preserved, and therefore the degree of $p_{i}$ can be maintained constant). This projector is employed in order to preserve the exact Toeplitz structure at each subsequent level of projection.

It is possible to preserve the exact Toeplitz structure at each level, cutting less information. In this paper we propose a different choice, i.e.,

(D) $P_{i+1}^{i}=K_{i+1}^{i}\{t\} T_{n_{i}}\left(p_{i}\right)$,

where $t$ is defined again as the degree of $p_{0}$ minus 1 (we remind the reader that the degree of $p_{i}$ is constant with respect to $i$ ), while

$$
K_{i+1}^{i}\{t\}=\left[0_{n_{i+1}-t}^{t}\left|K_{n_{i+1}-t}^{n_{i}-2 t}\right| 0_{n_{i+1}-t}^{t}\right] \in \mathbf{R}^{\left(n_{i+1}-t\right) \times n_{i}} .
$$

Where $0_{\alpha}^{\beta} \in \mathbb{R}^{\alpha \times \beta}$ is the null matrix and $K_{n_{i+1}-t}^{n_{i}-2 t} \in \mathbb{R}^{\left(n_{i+1}-t\right) \times\left(n_{i}-2 t\right)}$ is the usual cutting matrix where we put in evidence the dimensions instead of the recursion levels. We remark that, to apply the MGM recursively, we must start from dimension $n_{0}=2^{k_{0}}-1-2 t$; hence the dimension of problem at each sublevel is $n_{i}=2^{k_{0}-i}-1-2 t$. The matrix $K_{i+1}^{i}\{t\}$ is the cutting matrix that preserves the Toeplitzness at each level cutting the lowest possible level of information. Furthermore, we observe that $K_{i+1}^{i}[t]=K_{i+1}^{i}\{2 t\}$ for $t \geqslant 1$, and in addition, as can be experimentally verified, the number of iterations required by the MGM to reach a fixed precision is bounded from above by a constant independent of $n$ (optimality). However, the involved constant bound is much higher with the choice (C) than with the choice (D) and this is due to the quantity of information that we lose in the involved choices.

Analogously, in the multilevel case, $p_{i}$ is a suitable multivariate nonnegative polynomial of partial degrees $t_{i}+1$, with $i=1, \ldots, d$. Let $U_{i+1}^{i}\{t\}=K_{i+1}^{i}\left\{t_{1}\right\} \otimes \cdots \otimes$ $K_{i+1}^{i}\left\{t_{d}\right\}$, we define $P_{i+1}^{i}=U_{i+1}^{i}\{t\} T_{n_{i}}\left(p_{i}\right)$, where $n_{i}=\left(\left(n_{i}\right)_{1}, \ldots,\left(n_{i}\right)_{d}\right)$. Therefore, the $d$-level Toeplitz matrix at MGM recursion level $i+1$ is $T_{n_{i+1}}\left(f_{i+1}\right)=$ $P_{i+1}^{i} T_{n_{i}}\left(f_{i}\right)\left(P_{i+1}^{i}\right)^{H} \in \mathbb{R}^{N\left(n_{i+1}\right) \times N\left(n_{i+1}\right)}$ for $i=0, \ldots, m-1$, where each component of $n_{i+1}$ is defined as in the unilevel case.

The definition of the smoothing operators follows the same lines as in section 4.1. In [27] the TGM smoothing property is proved in the Toeplitz case and, by Remark 2.4, we can extend the same property to MGM.

7. Numerical experiments. In this section we present a wide numerical experimentation both in monodimensional and bidimensional cases. We stress that in both 
TABLE 7.1

Post-smoother strategies: $(a, b, c)$, where $a=$ iterations of Richardson with $\omega_{i}=1 / \max \left(f_{i}\right), b$ $=$ iterations of Richardson with $\omega_{i}=2 / \max \left(f_{i}\right), c=$ iterations of $C G ; i=0, \ldots, m-1$.

\begin{tabular}{|c||c|c|c|c|c|}
\hline & $(1,0,0)$ & $(2,0,0)$ & $(4,0,0)$ & $(1,1,0)$ & $(1,0,1)$ \\
\hline \hline$\rho\left(M G M_{0}\right)$ & 0.75 & 0.5625 & 0.3164 & 0.375 & nonstationary \\
\hline$\#$ iterations & 83 & 42 & 21 & 25 & 17 \\
\hline
\end{tabular}

situations we obtain similar results (iteration number independent of problem dimension), so our algorithm performances are not worse for multidimensional problems. In particular, this property is preserved also for the generalization of our algorithm to multilevel Toeplitz systems proposed in section 6 .

In what follows, the initial guess is $\mathbf{x}^{(0)}=\mathbf{0}$, the vector $\mathbf{b}$ is calculated from the exact solution $\mathbf{x}_{i}=i / n, i=1, \ldots, n$. The operations are executed in double precision and the termination condition is $\left\|\mathbf{r}_{0}^{(k)}\right\|_{2} \leqslant \varepsilon\|\mathbf{b}\|_{2}$, where $\varepsilon=10^{-11}$ in the monodimensional case and $\varepsilon=10^{-7}$ in the bidimensional case, since the high condition number does not allow a more accurate solution even in double precision. Concerning bidimensional problems, for simplicity, we consider the same dimensions in both directions. In the monodimensional V-cycle algorithm, the system at the coarsest level has dimension $\left(2^{3}-1\right) \times\left(2^{3}-1\right)$, while in the twodimensional case the size is $\left(2^{3}-1\right)^{2} \times\left(2^{3}-1\right)^{2}$.

7.1. On the smoother choice. Our theoretical analysis of convergence and optimality is done for only one iteration of a Richardson post-smoother with best parameter $\omega_{i}=1 / \max \left(f_{i}\right)$ for $i=0, \ldots, m-1$. Obviously, by increasing the number of iterations of the post-smoother or by adding a pre-smoother, the MGM converges more rapidly. In Table 7.1 we report the MGM spectral radius and the number of iterations when varying the post-smoother strategy. The problem dimension is not reported since by the MGM optimality the spectral radius and the number of iterations does not change for different dimensions. From this table we can see as the MGM spectral radius decreases, the number of iterations required by the method about halves when we double the number of iterations of the smoother. The latter behavior stresses the strength of our MGM, since, by doubling the number of smoothing steps, the overall cost of a single $\mathrm{V}$-cycle iteration is slightly less than doubled. Furthermore, from Table 7.1 we observe that the use of a multi-iterative strategy (see [28]) allows one to increase the MGM convergence speed: here for multi-iterative strategy we mean a fast iterative solver obtained by the combination of possibly slow basic iterations but with spectral complementary behavior. Indeed, one step of post-smoother with Richardson and $\omega_{i}=1 / \max \left(f_{i}\right)$ and one with $\omega_{i}=2 / \max \left(f_{i}\right), i=0, \ldots, m-1$, lead to a V-cycle iteration which is of the same cost as the one with two iterations of post-smoother with Richardson and $\omega_{i}=1 / \max \left(f_{i}\right)$ for $i=0, \ldots, m-1$ : however the number of iterations for reaching a given accuracy is roughly halved. According to this strategy, using the CG (not a stationary method!), the number of iterations is further reduced as reported in the last column of Table 7.1. We notice that the application of a constant number of CG steps is a nonstationary iteration which reduces to a specific Richardson method with varying parameter when we have only one CG step. The important observation is that both Richardson with $\omega_{i}=2 / \max \left(f_{i}\right)$ and one step (or a few steps) of the CG method are not smoothers but, according to the terminology of the multi-iterative methods, re intermediate (or residual) iterations. In actuality, in a V-cycle, the smoother "well approximates" the solution in the subspace 
where the coefficient matrix is well conditioned, the coarse grid correction (CGC) "well approximates" the solution in the ill-conditioned subspace (if the projector is properly chosen) and the intermediate or residual iteration takes care of the possible subspace where both the smoother and the CGC iterations failed to be effective: it is the spectral complementarity of these basic iterations that makes the whole multiiterative procedure fast (see [28]). In the specific case of a V-cycle, we observe that the action of the smoother and CGC is enough for obtaining an asymptotically optimal method and therefore the role of the intermediate iteration amounts to accelerating the global convergence speed (see also [31]).

According to the previous reasoning, in the following, when not differently specified, we have used one iteration of relaxed Richardson method with weight $\left\|f_{i}\right\|_{\infty}^{-1}$ as pre-smoother (a real smoother!) and one CG iteration as post-smoother (a residual iteration). Notice that the latter V-cycle has the same convergence features (see [31]) of a V-cycle with two steps of post-smoothing (one step of Richardson with weight $\left\|f_{i}\right\|_{\infty}^{-1}$ and one step of $\mathrm{CG}$ ): we stress that the combination of the former two basic iterations is a smoother, i.e., it satisfies the smoothing property in accordance with Proposition 4.1.

7.2. Elliptic PDEs. Let us consider a $d$-dimensional problem on the rectangular domain $\Omega=[0,1]^{d}$ :

$$
\left\{\begin{array}{l}
(-1)^{q} \sum_{i=1}^{d} \frac{\partial^{q}}{\partial x_{i}^{q}}\left(a(x) \frac{\partial^{q}}{\partial x_{i}^{q}} u(x)\right)=g(x), \quad x \in \Omega, q \geq 1, \\
\text { homogeneous B.C. on } \partial \Omega
\end{array}\right.
$$

where $x=\left(x_{1}, \ldots, x_{d}\right)$, when discretized on a uniform grid of $n=\left(n_{1}, \ldots, n_{d}\right)$ subintervals using centered finite difference of minimal precision order 2 , it leads to a multilevel band $N(n) \times N(n)$ linear system $A_{n} \mathbf{y}=\mathbf{b}$, that does not belong to the multilevel Toeplitz class unless $a(x)$ is a constant function. In that case $A_{n}=T_{n}\left(f^{(q)}\right)$, where

$$
f^{(q)}(x)=\sum_{i=1}^{d}\left[2-2 \cos \left(x_{i}\right)\right]^{q},
$$

from the condition (4.6) and its generalization to the multidimensional case, we can choose $p_{i}=p^{(q)}(x)$ with

$$
p^{(q)}(x)=\prod_{j=1}^{d}\left[2+2 \cos \left(x_{j}\right)\right]^{q}
$$

which allows us to obtain the optimality of our MGM when applied to a linear system $\tau_{n}\left(f^{(q)}\right) \mathbf{y}=\mathbf{b}$.

For the $\tau$ algebra, Table 7.2 shows the number of iterations of our MGM when increasing the dimension $n$ both in the monodimensional and bidimensional case ( $n=$ $\left.\left(n_{1}, n_{2}\right)\right)$. Concerning the circulant algebra, as already stressed, $\mathcal{C}_{n}\left(f^{(q)}\right)$ is singular because $f^{(q)}$ vanishes at the origin. Therefore, we solve the system $\widetilde{\mathcal{C}}_{n}\left(f^{(q)}\right) \mathbf{y}=\mathbf{b}$, where $\widetilde{\mathcal{C}}_{n}\left(f^{(q)}\right)$ is the stabilized version of $\mathcal{C}_{n}\left(f^{(q)}\right)$ defined in (1.5). Table 7.3 shows the number of iterations of our MGM applied to these systems in the $\tau$ algebra case.

We remark that our MGM shows an optimal behavior also in the multilevel case, and indeed a theoretic extension of the result reported in section 4 to the multidimensional context is reported in [2]. 
TABLE 7.2

Tau case: Number of iterations for increasing dimensions $N(n)$ both in the monodimensional case $(N(n)=n)$ and in the bidimensional case $\left(N(n)=n_{1} n_{2}, n_{1}=n_{2}\right)$.

\begin{tabular}{||c|c|c|c||c|c|c|c||}
\hline \hline \multicolumn{3}{||c||}{ 1D (monodimensional) } & \multicolumn{4}{|c||}{ 2D (bidimensional) } \\
\cline { 2 - 6 }$n$ & \multicolumn{3}{|c|}{ \# iterations } & \multirow{2}{*}{$n_{1} \cdot n_{2}$} & \multicolumn{3}{|c}{ \# iterations } \\
\cline { 2 - 6 } & $f^{(1)}$ & $f^{(2)}$ & $f^{(3)}$ & & $f^{(1)}$ & $f^{(2)}$ & $f^{(3)}$ \\
\hline \hline $2^{7}-1$ & 14 & 17 & 33 & $\left(2^{6}-1\right)^{2}$ & 11 & 20 & 37 \\
$2^{8}-1$ & 14 & 17 & 33 & $\left(2^{7}-1\right)^{2}$ & 11 & 20 & 37 \\
$2^{9}-1$ & 14 & 17 & 33 & $\left(2^{8}-1\right)^{2}$ & 10 & 20 & 37 \\
$2^{10}-1$ & 15 & 17 & 33 & $\left(2^{9}-1\right)^{2}$ & 10 & 20 & 36 \\
\hline \hline
\end{tabular}

TABLE 7.3

Circulant case with stabilization: Number of iterations for increasing dimensions $N(n)$ both in the monodimensional case $(N(n)=n)$ and in the bidimensional case $\left(N(n)=n_{1} n_{2}, n_{1}=n_{2}\right)$.

\begin{tabular}{|c|c|c|c|c|c|c|c|}
\hline \multicolumn{4}{|c|}{ 1D (monodimensional) } & \multicolumn{4}{|c|}{ 2D (bidimensional) } \\
\hline \multirow[b]{2}{*}{$n$} & \multicolumn{3}{|c|}{ \# iterations } & \multirow[b]{2}{*}{$n_{1} \cdot n_{2}$} & \multicolumn{3}{|c|}{ \# iterations } \\
\hline & $f^{(1)}$ & $f^{(2)}$ & $f^{(3)}$ & & $f^{(1)}$ & $f^{(2)}$ & $f^{(3)}$ \\
\hline $2^{7}$ & 13 & 17 & 31 & $\left(2^{6}\right)^{2}$ & 10 & 19 & 34 \\
\hline $2^{8}$ & 14 & 17 & 31 & $\left(2^{7}\right)^{2}$ & 10 & 19 & 34 \\
\hline $2^{9}$ & 14 & 17 & 31 & $\left(2^{8}\right)^{2}$ & 10 & 19 & 34 \\
\hline $2^{10}$ & 14 & 17 & 31 & $\left(2^{9}\right)^{2}$ & 10 & 19 & 34 \\
\hline
\end{tabular}

Through the procedure described in section 6 we can also directly solve the system $T_{n}\left(f^{(q)}\right) \mathbf{x}=\mathbf{b}$ : we must only take care to define the correct dimension to allow the MGM recursive application. In Table 7.4 the degree of $p_{i}$ is $\lceil(q+1) / 2\rceil$ instead of $q$ (refer to section 8), since the columns number (i.e., information) deleted from our algorithm is proportional to the degree of $p_{i}$. Furthermore, to recover a practically optimal behavior we perform $\nu_{i}=2+i$ iterations of pre-smoother and $\nu_{i}=2+i$ iterations of post-smoother at level $i$ (as proposed in [31]). According to the definition of the cutting matrix $K_{i+1}^{i}\{t\}$, to apply recursively the MGM, the dimension of the $i$ th projected system is $2^{r}-\xi$, where $r \in \mathbb{N}$ and $\xi=2\lceil(q+1) / 2\rceil-1$. From Table 7.4 we observe that our MGM shows again a practically optimal behavior both in monodimensional and bidimensional Toeplitz cases and, moreover, we stress that the cost of every MGM iteration with $\nu_{i}=2+i$ is still linear as the size $N(n)$ of the coefficient matrix (see the analysis of the computational cost in [31]).

7.3. Independency from the spectral decomposition of the solution. For our experimentation so far, we obtained the data vector $\mathbf{b}$ from the exact solution $\mathbf{x}_{i}=i / n, i=1, \ldots, n$ (initial solution $\mathbf{x}^{(0)}=\mathbf{0} \in \mathbb{R}^{n}$ ). Here we emphasize that the behavior of our algorithm does not depend on the particular spectral decomposition of the exact solution $\mathbf{x}$. We take four different types of solution where the coefficient matrix of the system is $\tau_{n}\left(f^{(2)}\right)$ and in Table 7.5 we report the iteration number required by the MGM to converge. From this table we observe a similar behavior for every different type of solution stressing the robustness of our algorithm.

7.4. Zero not in the origin. We present an example where the generating function $f_{0}$ does not vanish at the origin. More explicitly, the symbol

$$
f_{0}(x)=(1-\cos (x-1))(1-\cos (x+1))=(\cos (1)-\cos (x))^{2}
$$


TABLE 7.4

Toeplitz case: Number of iterations for increasing dimensions $N(n)$ both in the monodimensional case $(N(n)=n)$ and in the bidimensional case $\left(N(n)=n_{1} n_{2}, n_{1}=n_{2}\right)$ with $\xi=2\lceil(q+1) / 2\rceil-1, q=1,2,3$.

\begin{tabular}{||c|c|c|c||c|c|c|c||}
\hline \hline \multicolumn{3}{||c|}{ 1D (monodimensional) } & \multicolumn{4}{|c||}{ 2D (bidimensional) } \\
\cline { 2 - 4 }$n$ & \multicolumn{3}{|c|}{ \# iterations } & \multirow{2}{*}{$n_{1} \cdot n_{2}$} & \multicolumn{3}{|c|}{ \# iterations } \\
\cline { 2 - 5 } & $f^{(1)}$ & $f^{(2)}$ & $f^{(3)}$ & & $f^{(1)}$ & $f^{(2)}$ & $f^{(3)}$ \\
\hline \hline $2^{7}-\xi$ & 9 & 41 & 53 & $\left(2^{6}-\xi\right)^{2}$ & 6 & 24 & 33 \\
$2^{8}-\xi$ & 9 & 44 & 54 & $\left(2^{7}-\xi\right)^{2}$ & 6 & 26 & 33 \\
$2^{9}-\xi$ & 10 & 47 & 54 & $\left(2^{8}-\xi\right)^{2}$ & 6 & 27 & 33 \\
$2^{10}-\xi$ & 9 & 48 & 55 & $\left(2^{9}-\xi\right)^{2}$ & 6 & 29 & 33 \\
\hline \hline
\end{tabular}

TABLE 7.5

Different type of solution: Number of iterations for increasing dimension $n$ for $\tau_{n}\left(f^{(2)}\right)$.

\begin{tabular}{||c||c|c|c|c||}
\hline \hline \multirow{2}{*||}{$n$} & \multicolumn{4}{|c||}{ \# iterations for $\mathbf{x}_{i}=$} \\
\cline { 2 - 5 } & $\frac{i}{n}$ & $(-1)^{i}$ & $\cos \left(\frac{2 i \pi}{n}\right)$ & 1 \\
\hline \hline $2^{7}-1$ & 17 & 15 & 17 & 17 \\
$2^{8}-1$ & 17 & 14 & 17 & 17 \\
$2^{9}-1$ & 17 & 14 & 17 & 17 \\
$2^{10}-1$ & 17 & 14 & 17 & 17 \\
\hline \hline
\end{tabular}

is even and for $x \in[0,2 \pi)$ vanishes at 1 and $2 \pi-1$ with order 2 . For simplicity, we consider only the monodimensional $\tau$ algebra case, but the same considerations hold in the Circulant and Hartley algebras as well. According to (4.3), choosing

$$
p_{0}(x)=(\cos (1)+\cos (x))^{2}
$$

we have an optimal MGM for $\tau_{n}\left(f_{0}\right)$. Fixing $x_{0}^{(1)}=1$ and $x_{0}^{(2)}=2 \pi-1$, the position of the new zeros $x_{i}^{(k)}$ of $f_{i}$, for $i=0, \ldots, m-1$ with $k=1,2$, moves according to Proposition 4.5 and then the functions $p_{i}$ change at each level $i$. By applying the MGM, since we have two zeros of order two, we strengthen the smoothers by performing two iterations of pre-smoother and post-smoother. In Table 7.6 we report the number of iterations required for convergence, which is practically constant with regard to the dimension $n$, showing an optimal behavior in this case, too.

7.5. Image restoration problems. In the restoration of blurred images with Dirichlet boundary conditions we solve a system with coefficient matrix $T_{n}(f)$, where $f\left(x_{1}, x_{2}\right)$ is small and even indefinite when $x_{1}$ and $x_{2}$ approach $\pi$ (see also [6, 18]). Let $\mathcal{S}$ be the true image (for instance a "satellite") and let us consider the blurred image

$$
S=T_{n}\left(\psi\left(x_{1}, x_{2}\right)\left[4+2 \cos \left(x_{1}\right)+2 \cos \left(x_{2}\right)\right]^{3}\right) \mathcal{S}
$$

where the matrix $T_{n}\left(\psi\left(x_{1}, x_{2}\right)\left[4+2 \cos \left(x_{1}\right)+2 \cos \left(x_{2}\right)\right]^{3}\right)$ represents the compactly supported and spatially invariant "blurring operator." Here $\left[4+2 \cos \left(x_{1}\right)+2 \cos \left(x_{2}\right)\right]^{3}$ has a zero at $(\pi, \pi)$ of order 6 and $\psi\left(x_{1}, x_{2}\right)$ is a strictly positive polynomial with nonnegative Fourier coefficients: in this way the Fourier coefficients of $\psi\left(x_{1}, x_{2}\right)[4+$ 
TABLE 7.6

Zero not in the origin: Number of iterations increasing the dimension for $\tau_{n}\left((\cos (1)-\cos (x))^{2}\right)$.

\begin{tabular}{|c||c|c|c|c|}
\hline dimension & $2^{7}-1$ & $2^{8}-1$ & $2^{9}-1$ & $2^{10}-1$ \\
\hline \# iterations & 18 & 27 & 28 & 26 \\
\hline
\end{tabular}

TABLE 7.7

"Satellite" restoration: Error behavior in $\|\cdot\|_{2}$.

\begin{tabular}{|c||c|c|c|c|c|}
\hline \# iterations & 1 & 10 & 20 & 30 & 42 \\
\hline error norm & $8.271856 \mathrm{E}-01$ & $4.522643 \mathrm{E}-03$ & $4.490511 \mathrm{E}-04$ & $5.478008 \mathrm{E}-05$ & $4.781925 \mathrm{E}-06$ \\
\hline
\end{tabular}

$\left.2 \cos \left(x_{1}\right)+2 \cos \left(x_{2}\right)\right]^{3}$ are nonnegative as reported in the following mask:

$\begin{array}{ccccccccc}0 & 0 & 0 & 0 & 0.0002 & 0 & 0 & 0 & 0 \\ 0 & 0 & 0 & 0.0007 & 0.0033 & 0.0007 & 0 & 0 & 0 \\ 0 & 0 & 0.0010 & 0.0098 & 0.0260 & 0.0098 & 0.0010 & 0 & 0 \\ 0 & 0.0007 & 0.0098 & 0.0508 & 0.1022 & 0.0508 & 0.0098 & 0.0007 & 0 \\ 0.0002 & 0.0033 & 0.0260 & 0.1022 & 0.1829 & 0.1022 & 0.0260 & 0.0033 & 0.0002 \\ 0 & 0.0007 & 0.0098 & 0.0508 & 0.1022 & 0.0508 & 0.0098 & 0.0007 & 0 \\ 0 & 0 & 0.0010 & 0.0098 & 0.0260 & 0.0098 & 0.0010 & 0 & 0 \\ 0 & 0 & 0 & 0.0007 & 0.0033 & 0.0007 & 0 & 0 & 0 \\ 0 & 0 & 0 & 0 & 0.0002 & 0 & 0 & 0 & 0\end{array}$

Therefore, the associated Toeplitz sequence is asymptotically very ill-conditioned ( $[N(n)]^{3}$ ) and, despite this bad spectral behavior, the proposed multigrid method is optimal as emphasized by the linear convergence reported in Table 7.7. The considered choice is made in such a way that the resulting blur operator is a band approximation of the classical Gaussian blur whose Fourier coefficients are positive, symmetric and decay exponentially and whose generating function is close to zero in a neighborhood of $(\pi, \pi)$ and is positive elsewhere. Furthermore, the presence of the term $\psi\left(x_{1}, x_{2}\right)>0$ leads to a larger bandwidth so that the resulting blurring effect is more realistic.

As in the monodimensional case described in section 4.5 , in the multidimensional case also, our discretized integral problem is projected at the lower level into a discretized differential problem, so that the optimal behavior holds as shown in section 7.2 and Table 7.7. We consider the blurred image without noise and we solve the system (7.3) with the same smoother choice performed in section 7.2 for the Toeplitz case.

We stress that the regularization is not necessary since the image and the point spread function (PSF) are noise free and the conditioning of the blur operator is only polynomial with the size of the matrix. In Figure 7.1 we report the sequence of "satellite" image, the true image $\mathcal{S}$, the blurred image $S$, and the restored image with our MGM after 42 iterations.

Finally, we remark that in the case of noise the regularized systems $\left(T_{n}(f)+\right.$ $\mu I) \mathcal{S}=S$ with $\mu>0$ (see [6]) have a better conditioning than in the case of $\mu=0$ : therefore, our multigrid procedure, which is optimal for $\mu=0$, will be robust since the number of iterations will be bounded by a constant independent both of $N(n)$ and of $\mu>0$. In the first line of Table 7.8 we report the number of iterations for the restoration of the blurred satellite affected by $2 \%$ of noise with varying $\mu$ and with our projector. The second line of that table is obtained by using the same V-cycle with the same smoothers and with the classical projector used in the PDEs context [17] 


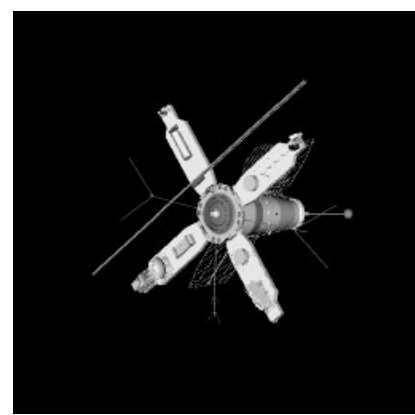

True Image (dim: $253 \times 253)$.

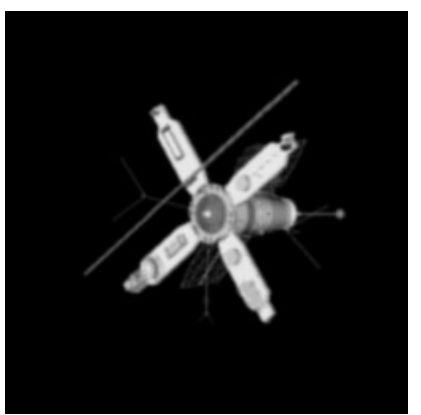

Blurred Image.

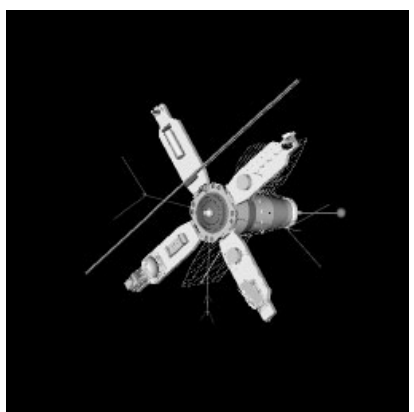

Restored Image after 42 iterations.

FIG. 7.1. Sequence of satellite images.

TABLE 7.8

Number of iterations for the satellite restoration with $2 \%$ of noise with varying $\mu$.

\begin{tabular}{|c||c|c|c|c|}
\hline$\mu$ & $10^{-1}$ & $10^{-2}$ & $10^{-3}$ & $10^{-4}$ \\
\hline \hline$p(x)=\left(2-2 \cos \left(x_{1}\right)\right)^{2}\left(2-2 \cos \left(x_{2}\right)\right)^{2}$ & 7 & 28 & 67 & 94 \\
\hline $\begin{array}{c}\text { our projector } \\
\text { linear interpolation }\end{array}$ & 7 & 32 & 216 & 1806 \\
\hline $\begin{array}{l}\left(2+2 \cos \left(x_{1}\right)\right)\left(2+2 \cos \left(x_{2}\right)\right) \\
\text { linter }\end{array}$ & 7 & & & \\
\hline
\end{tabular}

and by Huckle et al. [18] and R. Chan, T. Chan, and W. Wan [6] in image restoration: it is evident that our choice improves the convergence behavior substantially by maintaining the same computational cost. However, in the numerics with Gaussian blur in $[6,18]$ the authors obtained reasonably good results by applying the "wrong" prolongation operator: the reason is that, as smoothers, they used very sophisticated and costly solvers like PCG and FGMRES (flexible GMRES) with cosine/circulant preconditioners. Therefore the success of the whole procedure is mainly due to these auxiliary solvers which are reasonably effective on their own. Future work should try to combine their approach (with sophisticated smoothers) and the "correct" prolongation operators indicated in the present paper.

8. Concluding remarks, open problems, and future work. In this paper we have proposed a proof technique (based on matrix inequalities and on the PerronFrobenius theorem) which has been successful for a rigorous convergence analysis of the V-cycle procedure when applied to unilevel linear systems from algebras. We have also presented some algorithmic proposals for multilevel and Toeplitz structures: the numerical results (on discretized differential and integral problems) indicate an optimal convergence rate of our V-cycle procedures, but still we have to provide a theoretical analysis in the multilevel and Toeplitz settings.

Therefore future work should include the following directions:

- Toeplitz extension of the theory (for the TGM this has been done in [27]);

- multilevel extension of the theory (for matrix algebras see [2] while for multilevel Toeplitz structures only the TGM analysis is available [27]);

- multiple zero case (the TGM analysis and the numerical results are available $[7,15,16,18,27,31]$ : the MGM theory should be easy but tedious following the approach in the present paper).

Moreover, from an experimental viewpoint it is evident that conditions (2.15) are 
sufficient for the level independency and for the TGM optimality but they are not enough for the MGM optimality (see section 3). We have proven conditions (4.2) to be sufficient for the MGM optimality. However, from our numerics and from [31], we know that conditions (4.2) can be replaced by

$$
\begin{aligned}
\lim _{x \rightarrow x^{0}} \frac{p_{i}^{2}(\pi+x)}{f_{i}(x)} & =0, \\
p_{i}^{2}(x)+p_{i}^{2}(\pi+x) & >0 \quad \forall x
\end{aligned}
$$

by preserving the MGM optimal convergence rate (the latter are much weaker than (4.2) and just a little bit stronger than (2.15)!). Future work should also try to answer the previous question.

\section{REFERENCES}

[1] A. ARICò, Metodi Multigrid in Algebre Trigonometriche, BD thesis in Mathematics, University of Pisa, Pisa, Italy, 2002.

[2] A. Aricò and M. Donatelli, V-Cycle Optimal Convergence for Multilevel Structures, Numerische Mathematik, submitted, 2003.

[3] D. Bini and M. Capovani, Spectral and computational properties of band symmetric Toeplitz matrices, Linear Algebra Appl., 52/53 (1983), pp. 99-126.

[4] D. Bini And P. FAVATI, On a matrix algebra related to the discrete Hartley transform, SIAM J. Matrix Anal. Appl., 14 (1993), pp. 500-507.

[5] W. Briggs, V. Henson, And S. McCormick, A Multigrid Tutorial, 2nd ed., SIAM, Philadelphia, 2000.

[6] R.H. Chan, T.F. Chan, AND W. WAN, Multigrid for differential-convolution problems arising from image processing, in Scientific Computing, G. Golub, S.H. Lui, F. Luk, and R. Plemmons, eds., Springer-Verlag, Singapore, 1999, pp. 58-72.

[7] R.H. Chan, Q. Chang, and H. Sun, Multigrid method for ill-conditioned symmetric Toeplitz systems, SIAM J. Sci. Comput., 19 (1998), pp. 516-529.

[8] R.H. Chan, M. Donatelli, S. Serra-Capizzano, and C. Tablino Possio, Application of multigrid techniques to image restoration problems, Research Report CUHK-2002-14 (254), Department of Mathematics, The Chinese University of Hong Kong.

[9] R.H. Chan And M. NG, Conjugate gradient methods for Toeplitz systems, SIAM Rev., 38 (1996), pp. 427-482.

[10] R.H. Chan and G. Strang, Toeplitz equations by conjugate gradients with circulant preconditioner, SIAM J. Sci. Statist. Comput., 10 (1989), pp. 104-119.

[11] T.F. Chan, An optimal circulant preconditioner for Toeplitz systems, SIAM J. Sci. Statist. Comput., 9 (1988), pp. 766-771.

[12] F. Di Benedetto, Analysis of preconditioning techniques for ill-conditioned Toeplitz matrices, SIAM J. Sci. Comput., 16 (1995), pp. 682-697.

[13] F. Di Benedetto, G. Fiorentino, and S. Serra-Capizzano, C.G. preconditioning for Toeplitz matrices, Comput. Math. Appl., 25 (1993), pp. 35-45.

[14] M. Donatelli, Metodi Multigrid per Sistemi Lineari Strutturati Ed Applicazioni, BD thesis in Computer Science, University of Florence, 2002.

[15] G. Fiorentino and S. Serra-Capizzano, Multigrid methods for Toeplitz matrices, Calcolo, 28 (1991), pp. 283-305.

[16] G. Fiorentino and S. Serra-Capizzano, Multigrid methods for symmetric positive definite block Toeplitz matrices with nonnegative generating functions, SIAM J. Sci. Comput., 17 (1996), pp. 1068-1081.

[17] W. Hackbush, Multi-grid Methods and Applications, Springer-Verlag, New York, 1979.

[18] T. Huckle and J. Staudacher, Multigrid preconditioning and Toeplitz matrices, Electron. Trans. Numer. Anal., 13 (2002), pp. 81-105.

[19] D.G. Luenberger, Introduction to Dynamic Systems (Theory, Models 83 Applications), John Wiley \& Sons Inc., New York, 1979.

[20] M. NG, R. Chan AND W.C. TANG A fast algorithm for deblurring models with Neumann boundary conditions, SIAM J. Sci. Comput., 21 (1999), pp. 851-866.

[21] D. Noutsos, S. Serra-Capizzano, and P. Vassalos, Spectral equivalence and matrix algebra preconditioners for multilevel Toeplitz systems: A negative result, in Fast Algorithms for 
Structured Matrices: Theory and Applications, V. Olshevsky, ed., Contemp. Math. 323, AMS, Providence, RI, 2003, pp. 313-322.

[22] D. Noutsos, S. Serra-Capizzano, and P. Vassalos, Matrix algebra preconditioners for multilevel Toeplitz systems do not insure an optimal convergence rate, V. Pan ed., Theoret. Comp. Sci., to appear.

[23] J. W. Ruge and K. StüBen, Algebraic multigrid, in Multigrid Methods, Frontiers Appl. Math. 3, S.F. McCormick, ed., SIAM, Philadelphia, 1987, pp. 73-130.

[24] S. Serra-Capizzano, Proprietà Algebriche e Computazionali di Matrici di Toeplitz e Metodi Multigrid, BD thesis in Computer Science, University of Pisa, 1990.

[25] S. Serra-CaPizzano, Toeplitz preconditioners constructed from linear approximation processes,SIAM J. Matrix Anal. Appl., 20 (1998), pp. 446-465.

[26] S. Serra-Capizzano, Matrix algebra preconditioners for multilevel Toeplitz matrices are not superlinear, Linear Algebra Appl., 343/344 (2002), pp. 303-319.

[27] S. Serra-Capizzano, Convergence analysis of two-grid methods for elliptic Toeplitz and PDEs matrix-sequences, Numer. Math., 92 (2002), pp. 433-465.

[28] S. Serra-Capizzano, Multi-iterative methods, Comput. Math. Appl., 26 (1993), pp. 65-87.

[29] S. Serra-CapizzAno, A note on anti-reflective boundary conditions and fast deblurring models, SIAM J. Sci. Comput., 25 (2003), pp. 1307-1325.

[30] S. Serra-Capizzano and C. Tablino Possio, Spectral and structural analysis of high precision finite difference matrices for elliptic operators, Linear Algebra Appl., 293 (1999), pp. 85131.

[31] S. Serra-Capizzano and C. Tablino Possio, Multigrid methods for multilevel circulant matrices, SIAM J. Sci. Comput., to appear.

[32] S. Serra-Capizzano and E. Tyrtyshnikov, Any circulant-like preconditioner for multilevel matrices is not superlinear, SIAM J. Matrix Anal. Appl., 21 (1999), pp. 431-439.

[33] S. Serra-Capizzano and E. Tyrtyshnikov, How to prove that a preconditioner cannot be superlinear, Math. Comp., 72 (2003), pp. 1305-1316.

[34] E. Tyrtyshnikov, Circulant preconditioners with unbounded inverse, Linear Algebra Appl., 216 (1995), pp. 1-23.

[35] E. Tyrtyshnikov, A unifying approach to some old and new theorems on distribution and clustering, Linear Algebra Appl., 232 (1996), pp. 1-43.

[36] R.S. VArga, Matrix Iterative Analysis, Prentice Hall, Englewood Cliffs, NJ, 1962.

[37] H. Yserentant, Old and new convergence proofs for multigrid methods, Acta Numer., 2 (1993), pp. 285-326. 